\title{
Pseudo-Codeword Analysis of Tanner Graphs from Projective and Euclidean Planes*
}

\author{
Roxana Smarandache ${ }^{\dagger}$ and Pascal O. Vontobel ${ }^{\ddagger}$
}

\begin{abstract}
In order to understand the performance of a code under maximum-likelihood (ML) decoding, one studies the codewords, in particular the minimal codewords, and their Hamming weights. In the context of linear programming (LP) decoding, one's attention needs to be shifted to the pseudo-codewords, in particular to the minimal pseudocodewords, and their pseudo-weights. In this paper we investigate some families of codes that have good properties under LP decoding, namely certain families of low-density parity-check (LDPC) codes that are derived from projective and Euclidean planes: we study the structure of their minimal pseudo-codewords and give lower bounds on their pseudo-weight.
\end{abstract}

Index terms - Linear programming decoding, message-passing iterative decoding, minimal codewords, minimal pseudo-codewords, pseudo-weight, codes from projective planes, codes from Euclidean planes, pseudo-weight spectra.

\section{Introduction}

The motivation of this paper to look at minimal codewords and minimal pseudo-codewords is twofold. On the one hand we would like to give performance guarantees on linear programming (LP) decoding [1, 2], which is a decoding method that has recently emerged as an interesting approach to decoding codes. On the other hand, the connection made by Koetter and Vontobel [3, 4, 5] between message-passing iterative (MPI) decoding and LP decoding suggests that results for LP decoding have immediate implications for MPI decoding.

Apart from presenting some bounds on pseudo-weights that hold in general, this paper focuses solely on certain families of codes based on projective and Euclidean planes. One of

${ }^{*}$ The first author was partially supported by NSF Grant ITR-0205310. The second author was supported by NSF Grants ATM-0296033 and DOE SciDAC and by ONR Grant N00014-00-1-0966. The material in this paper has been presented in part at the 2005 International Symposium on Information Theory, Adelaide, Australia, Sep. 4-9, 2005, and at the 43rd Annual Allerton Conference on Communications, Control, and Computing, Monticello, IL, USA, Sep. 28-30.

${ }^{\dagger}$ Department of Mathematics and Statistics, San Diego State University, San Diego, CA 92182, USA. On leave at Department of Mathematics, University of Notre Dame, Notre Dame, IN 46556, USA. Email: rsmarand@nd.edu.

${ }^{\ddagger}$ Department of EECS, Massachusetts Institute of Technology, 77 Massachusetts Avenue, Cambridge, MA 02139, USA. Part of the work was done while being with the ECE Department, University of WisconsinMadison, 1415 Engineering Drive, Madison, WI 53706, USA. Email: pascal.vontobel@ieee.org. P.O.V. is the corresponding author. 
the reasons for this choice is that in the past, several groups of authors have experimentally observed that such codes can perform very well under MPI decoding, see e.g. [6] 7], and therefore these families of codes are a worthwhile object of study for making some first steps towards a rigorous understanding of the observed behavior. Another reason is that these families of codes have concise descriptions and large automorphism groups which may potentially be used to simplify their analysis.

The rest of this paper is structured as follows. In Sec. 2 we will introduce the two main families of codes under investigation. After having reviewed maximum liklihood (ML) decoding and LP decoding in Sec. 3] in Secs. 4and 5 we will explain why minimal codewords and minimal pseudo-codewords are important in the understanding of the performance of ML and LP decoding, respectively. Sec. 6] will present pseudo-weight spectra of some selected codes based on finite geometries. Then, in Sec. 7 we will analyze the possible pseudo-weights of pseudo-codewords and we will give upper and lower bounds on the pseudoweight of certain vectors. In Sec. 8 we introduce the concept of effective minimal pseudocodewords and in Sec. 9 we study the structure of minimal pseudo-codewords. Finally, in Sec. 10 we offer some conclusions.

In the following, $\mathbb{R}, \mathbb{R}_{+}$, and $\mathbb{R}_{++}$will be the set of real numbers, the set of non-negative real numbers, and the set of positive real numbers, respectively. Moreover, the support of a vector $\mathbf{x}$ will be defined as $\operatorname{supp}(\mathbf{x}) \triangleq\left\{i \mid x_{i} \neq 0\right\}$, the Hamming weight of a vector will be as usual $w_{\mathrm{H}}(\mathbf{x}) \triangleq|\operatorname{supp}(\mathbf{x})|$, and $\langle\mathbf{a}, \mathbf{b}\rangle \triangleq \sum_{i} a_{i} b_{i}$ will denote the standard inner product of two vectors of equal length.

\section{The Code Families under Investigation}

The codes under investigation come from the families of codes that were called type-I PGLDPC and type-I EG-LDPC codes in 7. Type-I PG-LDPC codes are defined as follows. Let $q \triangleq 2^{s}$ for some positive integer $s$ and consider a (finite) projective plane $\mathrm{PG}(2, q)$ (see e.g. [8, 9]) with $q^{2}+q+1$ points and $q^{2}+q+1$ lines: each point lies on $q+1$ lines and each line contains $q+1$ points. ${ }^{1}$ A standard way of associating a parity-check matrix $\mathbf{H}$ of a binary linear code to a finite geometry is to let the set of points correspond to the columns of $\mathbf{H}$, to let the set of lines correspond to the rows of $\mathbf{H}$, and finally to define the entries of $\mathbf{H}$ according to the incidence structure of the finite geometry. In this way, we can associate to the projective plane $\mathrm{PG}(2, q)$ the code $\mathcal{C}_{\mathrm{PG}(2, q)}$ with parity-check matrix $\mathbf{H} \triangleq \mathbf{H}_{\mathrm{PG}(2, q)}$, whose parameters are:

$\begin{array}{ll}\text { length } & n=q^{2}+q+1, \\ \text { dimension } & k=n-3^{s}-1, \\ \text { minimum Hamming distance } & d_{\text {min }}=q+2, \\ \text { uniform column weight of } \mathbf{H} & w_{\text {col }}=q+1, \\ \text { uniform row weight of } \mathbf{H} & w_{\text {row }}=q+1, \\ \text { size of } \mathbf{H} & n \times n .\end{array}$

In the usual way, we associate a Tanner graph $\mathbf{T}\left(\mathbf{H}_{\mathrm{PG}(2, q)}\right)$, 10, to the parity-check matrix $\mathbf{H}_{\mathrm{PG}(2, q)}$ : this graph consists of $n=q^{2}+q+1$ variable nodes of degree $w_{\text {col }}=q+1$ and of $n=q^{2}+q+1$ check nodes of degree $w_{\text {row }}=q+1$.

\footnotetext{
${ }^{1}$ Note that the "2" in $\operatorname{PG}(2, q)$ stands for the dimensionality of the geometry, which in the case of planes is 2 .
} 
Type-I EG-LDPC codes are defined as follows. Let $q \triangleq 2^{s}$ for some positive integer $s$ and consider a (finite) Euclidean plane $\operatorname{EG}(2, q)$ (see e.g. [8, 9]) with $q^{2}$ points and $q^{2}+q$ lines: each point lies on $q+1$ lines and each line contains $q$ points. We essentially use the same procedure as outlined above in order to associate a parity-check matrix to a finite geometry. But before doing this, we modify the Euclidean plane slightly: we select a point of $\mathrm{EG}(2, q)$ and remove it together with the $q+1$ lines through it. Doing so, we obtain an $\mathrm{EG}(2, q)$-based code $\mathcal{C}_{\mathrm{EG}(2, q)}$ with parity-check matrix $\mathbf{H} \triangleq \mathbf{H}_{\mathrm{EG}(2, q)}$, whose parameters are:

$\begin{array}{ll}\text { length } & n=q^{2}-1, \\ \text { dimension } & k=n-3^{s}+1, \\ \text { minimum Hamming distance } & d_{\text {min }}=q+1, \\ \text { uniform column weight of } \mathbf{H} & w_{\text {col }}=q, \\ \text { uniform row weight of } \mathbf{H} & w_{\text {row }}=q, \\ \text { size of } \mathbf{H} & n \times n .\end{array}$

Again, we can associate a Tanner graph $\mathrm{T}\left(\mathbf{H}_{\mathrm{EG}(2, q)}\right)$, [10, to the parity-check matrix $\mathbf{H}_{\mathrm{EG}(2, q)}$ : this graph consists of $n=q^{2}-1$ variable nodes of degree $w_{\text {col }}=q$ and of $n=q^{2}-1$ check nodes of degree $w_{\text {row }}=q$.

Both families of codes have the nice property that, with an appropriate ordering of the columns and rows, the parity-check matrices are circulant matrices, meaning that $\mathcal{C}_{\mathrm{PG}(2, q)}$ and $\mathcal{C}_{\mathrm{EG}(2, q)}$ are cyclic codes. This fact can e.g. be used for efficient encoding. Such symmetries can also substantially simplify the analysis. Note that the automorphism groups of $\mathcal{C}_{\mathrm{PG}(2, q)}$ and $\mathcal{C}_{\mathrm{EG}(2, q)}$ contain many more automorphisms besides the cyclic-shiftautomorphism implied by the cyclicity of the codes.

\section{$3 \quad$ ML and LP Decoding}

In this section we briefly review ML and LP decoding [1, 2]. Consider a binary linear code $\mathcal{C} \subseteq\{0,1\}^{n}$ of length $n$ and dimension $k$ that is used for data communication over a memoryless binary-input channel with channel law $p_{Y \mid X}(y \mid x)$. The transmitted codeword will be called $\mathbf{x} \triangleq\left(x_{1}, \ldots, x_{n}\right)$ and the received vector will be called $\mathbf{y} \triangleq\left(y_{1}, \ldots, y_{n}\right)$. Based on the received vector, we can define the log-likelihood ratio (LLR) vector $\boldsymbol{\lambda} \triangleq\left(\lambda_{1}, \ldots, \lambda_{n}\right) \in$ $(\mathbb{R} \cup\{ \pm \infty\})^{n}$ to be the vector containing the LLRs $\lambda_{i} \triangleq \log \left(p_{Y \mid X}\left(y_{i} \mid 0\right) / p_{Y \mid X}\left(y_{i} \mid 1\right)\right), i=$ $1, \ldots, n$. Using the canonical embedding of the set $\{0,1\}$ into $\mathbb{R}$ and of the set $\mathcal{C}$ into $\mathbb{R}^{n}$, ML decoding can then be cast as

$$
\hat{\mathbf{x}} \triangleq \arg \min _{\mathbf{x} \in \mathcal{C}}\langle\mathbf{x}, \boldsymbol{\lambda}\rangle
$$

Letting $\operatorname{conv}(\mathcal{C})$ be the convex hull of $\mathcal{C}$ in $\mathbb{R}^{n}$, the above ML decoding rule can also be formulated as

$$
\hat{\mathbf{x}} \triangleq \arg \min _{\mathbf{x} \in \operatorname{conv}(\mathcal{C})}\langle\mathbf{x}, \boldsymbol{\lambda}\rangle .
$$

Unfortunately, for most codes of interest, the description complexity of $\operatorname{conv}(\mathcal{C})$ grows exponentially in the block length and therefore finding the minimum in (2) with a linear programming solver is highly impractical for reasonably long codes. ${ }^{2}$

\footnotetext{
${ }^{2}$ Exceptions to this observation include for example the class of convolutional codes with not too many states.
} 
A standard approach in optimization theory and practice is to replace a minimization problem by a relaxed minimization problem, in our case we replace the minimization over $\operatorname{conv}(\mathcal{C})$ by a minimization over some easily describable polytope $\mathcal{P}$ which is a relaxation of $\operatorname{conv}(\mathcal{C})$ :

$$
\hat{\mathbf{x}} \triangleq \arg \min _{\mathbf{x} \in \mathcal{P}}\langle\mathbf{x}, \boldsymbol{\lambda}\rangle .
$$

If $\mathcal{P}$ is strictly larger than $\operatorname{conv}(\mathcal{C})$ then the decision rule in (3) obviously represents a suboptimal decoder. A relaxation that works particularly well for LDPC codes is given by the following approach 1, 2. Let $\mathcal{C}$ be described by an $m \times n$ parity-check matrix $\mathbf{H}$ with rows $\mathbf{h}_{1}, \mathbf{h}_{2}, \ldots, \mathbf{h}_{m}$. Then the polytope $\mathcal{P} \triangleq \mathcal{P}(\mathbf{H})$, in this context also called the fundamental polytope [3, 4, is defined as

$$
\mathcal{P} \triangleq \bigcap_{j=1}^{m} \operatorname{conv}\left(\mathcal{C}_{j}\right) \quad \text { with } \quad \mathcal{C}_{j} \triangleq\left\{\mathbf{x} \in\{0,1\}^{n} \mid\left\langle\mathbf{h}_{j}, \mathbf{x}\right\rangle=0 \bmod 2\right\}
$$

Note that $\mathcal{P}$ is a convex set within $[0,1]^{n}$ that contains $\operatorname{conv}(\mathcal{C})$, but whose description complexity is typically much smaller than the one of $\operatorname{conv}(\mathcal{C})$. Points in the set $\mathcal{P}$ will be called pseudo-codewords. Because the set $\mathcal{P}$ is usually strictly larger than $\operatorname{conv}(\mathcal{C})$, it can obviously happen that the decoding rule in (3) delivers a vertex of $\mathcal{P}$ that is not a codeword. Such vertices that correspond to pseudo-codewords that are not codewords are the reason for the sub-optimality of LP decoding (cf. [1, 2, 3, 4]). Note that $\mathcal{P}=\mathcal{P}(\mathbf{H})$ is a function of the parity-check matrix $\mathbf{H}$ that describes the code $\mathcal{C}$. Different parity-check matrices for the same code might therefore lead to different fundamental polytopes. It is worthwhile to remark though that all these fundamental polytopes have the property that $\mathcal{P}(\mathbf{H}) \cap\{0,1\}^{n}=\mathcal{C}$, i.e. all points of $\mathcal{P}(\mathbf{H})$ with integral coordinates are also codewords [1, 2] .

\section{Minimal Codewords}

In this section we will discuss minimal codewords and explain their importance with respect to ML decoding. Although ML decoding is often impractical, knowing bounds on the block error rate of an ML decoder can help in assessing the performance of sub-optimal but practical decoding algorithms.

Definition 1 Let $\mathcal{C}$ be a linear code. A non-zero codeword $\mathbf{x} \in \mathcal{C}$ is called minimal if and only if its support does not (strictly) contain the support of any other non-zero codeword as a proper subset. The set of all minimal codewords of $\mathcal{C}$ is commonly denoted by $\mathcal{M}(\mathcal{C})$.

We will henceforth assume that we transmit a binary linear code $\mathcal{C}$ over a binary-input output-symmetric channel (cf. e.g. [11, Def. 1]). For this setup, when studying the ML decoder in (1) or (2), we can without loss of generality assume that the zero codeword was sent, because all decision regions are congruent. The importance of minimal codewords lies in the following considerations.

Theorem 2 (cf. e.g. [12]) Let $\mathcal{C}$ be a binary linear code of length $n$ and for $\mathbf{x} \in \mathcal{C}$ let

$$
\mathcal{D}_{\mathbf{x}}^{\mathrm{ML}} \triangleq\left\{\boldsymbol{\lambda} \in \mathbb{R}^{n} \mid\left\langle\mathbf{x}^{\prime}, \boldsymbol{\lambda}\right\rangle \geqslant\langle\mathbf{x}, \boldsymbol{\lambda}\rangle \text { for all } \mathbf{x}^{\prime} \in \mathcal{C} \backslash\{\mathbf{x}\}\right\}
$$


be the region in the LLR space where the $M L$ decoder decides in favor of the codeword $\mathbf{x} .^{3}$ Then the decision region $\mathcal{D}_{\mathbf{x}}^{\mathrm{ML}}$ of a codeword $\mathbf{x} \in \mathcal{C}$ shares a facet ${ }^{4}$ with the decision region $\mathcal{D}_{\mathbf{0}}^{\mathrm{ML}}$ of the zero codeword if and only if $\mathbf{x}$ is a minimal codeword.

Therefore, knowing the minimal codewords of the code $\mathcal{C}$ is sufficient in order to assess its ML decoding performance. In the following, we mention a few known facts about minimal codewords of codes that helped us in our investigations in the later chapters of this paper.

Lemma $3([14,15])$ Let $\mathcal{M}(\mathcal{C})$ denote the set of all minimal codewords of a binary $[n, k, d]$ code $\mathcal{C}$. Then:

1. $\mathcal{M}(\mathcal{C})$ spans $\mathcal{C}$.

2. If $\mathbf{H}(\mathcal{U})$ denotes the submatrix of $\mathbf{H}$ with columns indexed by the set $\mathcal{U} \subseteq\{1,2, \ldots, n\}$, we have that a codeword $\mathbf{x} \in \mathcal{C}$ is minimal if and only if $\operatorname{rank}(\mathbf{H}(\operatorname{supp}(\mathbf{x})))=$ $|\operatorname{supp}(\mathbf{x})|-1=w_{\mathrm{H}}(\mathbf{x})-1$.

3. If $\mathbf{x} \in \mathcal{M}(\mathcal{C})$ then $|\operatorname{supp}(\mathbf{x})|=w_{\mathrm{H}}(\mathbf{x}) \leqslant n-k+1$.

4. If $\mathbf{x} \in \mathcal{C}$ and $|\operatorname{supp}(\mathbf{x})|=w_{\mathrm{H}}(\mathbf{x}) \leqslant 2 d-1$ then $\mathbf{x} \in \mathcal{M}(\mathcal{C})$.

5. Every non-minimal codeword can be written as a sum of two non-zero codewords with disjoint supports.

Let us mention some further results about minimal codewords (here we also include non-binary codes). The problem of classifying the minimal codewords is completely solved only for $q$-ary Hamming codes, for the second order binary Reed-Muller codes $\operatorname{RM}(2, m)$, and for the $\mathbb{Z}_{4}$ Kerdock codes [15, 16, 17, and partially for the binary Reed-Muller code of order $r, \operatorname{RM}(r, m)$ [18. However, in general it seems to be quite difficult to describe the set of minimal codewords for an arbitrary linear code even in the binary case.

We remark that in the context of linear codes, Hwang [14] was the first to consider the set of minimal codewords of a code (there called the "projecting set of a code"). He studied them in connection with two modifications of the correlation decoding algorithm. ${ }^{5}$

Minimal codewords and their properties arise also in connection with secret sharing. As it was first pointed out in [17, minimal vectors in a linear code completely specify the access structure of the linear secret sharing scheme defined by the code. This line of research was pursued in [15, 16]. We finally note that minimal vectors were also studied in combinatorics under the concept of cycles of linear matroids.

\section{The Fundamental Cone, Minimal Pseudo-Codewords, and Spectra}

In this section we will shift our attention to LP decoding and the objects of interest: pseudocodewords and in particular the minimal pseudo-codewords. For LP decoding of a binary linear code that is used for data transmission over a binary-input output-symmetric channel, it is sufficient to consider the part of the fundamental polytope $\mathcal{P}$ around the vertex $\mathbf{0}$,

\footnotetext{
${ }^{3}$ We assume that during ML decoding ties between decoding regions are resolved randomly.

${ }^{4} \mathrm{~A}$ facet is an $n-1$ dimensional face of a polytope, see e.g. [13.

${ }^{5}$ In the light of the ML decoder as formulated in (2), these algorithms can be seen as variations of the simplex method (cf. e.g. 19]) that minimizes $\langle\mathbf{x}, \boldsymbol{\lambda}\rangle$ over the polytope $\operatorname{conv}(\mathcal{C})$.
} 
cf. [3, 4, i.e. the fundamental cone. (See also [1, 2] that discuss this so-called "C $\mathcal{C}$-symmetry" property).

Lemma $4([\mathbf{1}, \mathbf{2}, \mathbf{4}])$ Let $\mathcal{C}$ be an arbitrary binary linear code and let $\mathbf{H}$ be its parity-check matrix. We let $\mathcal{J} \triangleq \mathcal{J}(\mathbf{H})$ be the set of row indices of $\mathbf{H}$ and we let $\mathcal{I} \triangleq \mathcal{I}(\mathbf{H})$ be the set of column indices of $\mathbf{H}$, respectively. For each $j \in \mathcal{J}$, we let $\mathcal{I}_{j} \triangleq \mathcal{I}_{j}(\mathbf{H}) \triangleq\left\{i \in \mathcal{I} \mid h_{j i}=1\right\}$. Let the fundamental cone $\mathcal{K}(\mathbf{H})$ of $\mathbf{H}$ be the conic hull of the fundamental polytope $\mathcal{P}(\mathbf{H})$. Then, $\mathcal{K}(\mathbf{H})$ is the set of vectors $\boldsymbol{\omega} \in \mathbb{R}^{n}$ that satisfy

$$
\begin{aligned}
\forall j \in \mathcal{J}, \forall i \in \mathcal{I}_{j}: & \sum_{i^{\prime} \in \mathcal{I}_{j} \backslash\{i\}} \omega_{i^{\prime}} \geqslant \omega_{i}, \\
\forall i \in \mathcal{I}: & \omega_{i} \geqslant 0 .
\end{aligned}
$$

We note that if $\boldsymbol{\omega}$ is in $\mathcal{K}(\mathbf{H})$, then also $\alpha \cdot \boldsymbol{\omega}$ is in $\mathcal{K}(\mathbf{H})$ for any $\alpha \in \mathbb{R}_{++}$. Moreover, for any $\boldsymbol{\omega}$ in $\mathcal{K}(\mathbf{H})$ there exists an $\alpha \in \mathbb{R}_{++}$(in fact, a whole interval of $\alpha$ 's) such that $\alpha \cdot \boldsymbol{\omega}$ is in $\mathcal{P}(\mathbf{H})$.

Let $\mathcal{D}_{\mathbf{0}}^{\mathrm{LP}} \triangleq\left\{\boldsymbol{\lambda} \in \mathbb{R}^{n} \mid\langle\boldsymbol{\omega}, \boldsymbol{\lambda}\rangle \geqslant 0\right.$ for all $\left.\boldsymbol{\omega} \in \mathcal{P}(\mathbf{H}) \backslash\{\boldsymbol{0}\}\right\}$ be the region where the LP decoder decides in favor of the codeword $\mathbf{0}{ }^{6}$ It can easily been seen that $\mathcal{D}_{\mathbf{0}}^{\mathrm{LP}}=\{\boldsymbol{\lambda} \in$ $\mathbb{R}^{n} \mid\langle\boldsymbol{\omega}, \boldsymbol{\lambda}\rangle \geqslant 0$ for all $\left.\boldsymbol{\omega} \in \mathcal{K}(\mathbf{H})\right\}$. Therefore, when studying LP decoding it is enough to know $\mathcal{K}(\mathbf{H})$; all vectors $\boldsymbol{\omega} \in \mathcal{K}(\mathbf{H})$ will henceforth be called pseudo-codewords. Moreover, two pseudo-codewords where one is a positive multiple of the other will be considered to be equivalent.

A class of pseudo-codewords that will be used a few times later on is the class of so-called unscaled pseudo-codewords 20, 4. These pseudo-codewords have integer entries and are derived from codewords in finite covers of the Tanner graph $\mathrm{T}(H)$. An important property of an unscaled pseudo-codeword $\boldsymbol{\omega}$ is that $\boldsymbol{\omega}(\bmod 2) \in \mathcal{C} .{ }^{7}$

Another important class of pseudo-codewords is the class of so-called minimal pseudocodewords:

Definition $5([3,4])$ Let $\mathcal{C}$ be an arbitrary binary linear code described by the parity-check matrix $\mathbf{H}$ whose fundamental cone is $\mathcal{K}(\mathbf{H})$. A vector $\boldsymbol{\omega} \in \mathcal{K}(\mathbf{H})$ is called a minimal pseudocodeword if the set $\left\{\alpha \cdot \boldsymbol{\omega} \mid \alpha \in \mathbb{R}_{+}\right\}$is an edge of $\mathcal{K}(\mathbf{H})$. The set of all minimal codewords will be called $\mathcal{M}_{\mathrm{p}}(\mathcal{K}(\mathbf{H})){ }^{8}$

For a given binary linear code $\mathcal{C}$ with parity-check matrix $\mathbf{H}$, the importance of the set $\mathcal{M}_{\mathrm{p}}(\mathcal{K}(\mathbf{H})$ ) lies in the following fact. From basic cone properties (cf. e.g. 21]), it can easily be seen that $\mathcal{D}_{\mathbf{0}}^{\mathrm{LP}}=\left\{\boldsymbol{\lambda} \in \mathbb{R}^{n} \mid\langle\boldsymbol{\omega}, \boldsymbol{\lambda}\rangle \geqslant 0\right.$ for all $\left.\boldsymbol{\omega} \in \mathcal{M}_{\mathrm{p}}(\mathcal{K}(\mathbf{H}))\right\}$. Therefore, the set $\mathcal{M}_{\mathrm{p}}(\mathcal{K}(\mathbf{H}))$ completely characterizes the behavior of the LP decoder. It can be shown [4] that for any minimal pseudo-codeword $\boldsymbol{\omega}$ there is an $\alpha \in \mathbb{R}_{++}$such that $\alpha \boldsymbol{\omega}$ is an unscaled pseudo-codeword, which, among other things, implies that all components of $\alpha \boldsymbol{\omega}$ are integers.

Note that the above notion of minimal pseudo-codewords generalizes the notion of minimal codewords in the following sense: whereas minimal pseudo-codewords correspond oneto-one to the edges of $\mathcal{P}(\mathbf{H})$ (or $\mathcal{K}(\mathbf{H})$ ) around $\mathbf{0}$, the minimal codewords correspond one-

\footnotetext{
${ }^{6}$ We assume that during LP decoding ties between decoding regions are resolved randomly.

${ }^{7}$ See [20] 4] for an exact definition of unscaled pseudo-codewords and their properties.

${ }^{8}$ Note that this definition implies that $\mathbf{0} \notin \mathcal{M}_{\mathrm{p}}(\mathcal{K}(\mathbf{H}))$.
} 
to-one to the edges of $\operatorname{conv}(\mathcal{C})$ around $\mathbf{0}$. (Minimal codewords are usually also minimal pseudo-codewords, but not always, as was remarked in [4.)

Because of the one-to-one relationship between parity-check matrices and Tanner graphs, the fundamental cone can also be seen as a function of the Tanner graph representing a code. Therefore, in order to emphasize the dependence of minimal pseudo-codewords on the representation of the code, we will sometimes talk about the minimal pseudo-codewords of a Tanner graph.

The fundamental cone is independent of the specific memoryless binary-input channel through which we are transmitting, however, the influence of a pseudo-codeword on the LP decoding performance depends very much on what channel is used. This influence will be measured by a channel-dependent pseudo-weight of pseudo-codewords; these pseudo-weights can be seen as generalizations of the Hamming weight that has traditionally been used to assess the performance of a code under ML decoding.

Definition 6 Let $\boldsymbol{\omega} \in \mathbb{R}_{+}^{n}$. The binary-input additive white Gaussian noise channel (AWGNC) pseudo-weight [22, [23, 4] of $\boldsymbol{\omega}$ is defined to be

$$
w_{\mathrm{p}}^{\mathrm{AWGNC}}(\boldsymbol{\omega}) \triangleq \frac{\|\boldsymbol{\omega}\|_{1}^{2}}{\|\boldsymbol{\omega}\|_{2}^{2}}
$$

if $\boldsymbol{\omega} \neq \mathbf{0}$ and $w_{\mathrm{p}}^{\mathrm{AWGNC}}(\boldsymbol{\omega}) \triangleq 0$ otherwise, where $\|\boldsymbol{\omega}\|_{1}$ and $\|\boldsymbol{\omega}\|_{2}$ are the $\mathcal{L}_{1}$ - and $\mathcal{L}_{2}$-norm of $\boldsymbol{\omega}$, respectively. Let $\boldsymbol{\omega}^{\prime} \in \mathbb{R}_{+}^{n}$ be a vector with the same components as $\boldsymbol{\omega}$ but in nonincreasing order. Introducing

$$
\begin{aligned}
f(\xi) & \triangleq \omega_{i}^{\prime} \quad(i-1<\xi \leqslant i, 0<\xi \leqslant n), \\
F(\xi) & \triangleq \int_{0}^{\xi} f\left(\xi^{\prime}\right) \mathrm{d} \xi^{\prime}, \\
e & \triangleq F^{-1}\left(\frac{F(n)}{2}\right),
\end{aligned}
$$

the BSC pseudo-weight [23, 4] is defined to be $w_{\mathrm{p}}^{\mathrm{BSC}}(\boldsymbol{\omega}) \triangleq 2$ if $\boldsymbol{\omega} \neq \mathbf{0}$ and $w_{\mathrm{p}}^{\mathrm{BSC}}(\boldsymbol{\omega}) \triangleq 0$ otherwise. Finally, the BEC pseudo-weight [23, 4] is defined to be

$$
w_{\mathrm{p}}^{\mathrm{BEC}}(\boldsymbol{\omega})=|\operatorname{supp}(\boldsymbol{\omega})| .
$$

Note that for $\mathbf{x} \in\{0,1\}^{n}$ we have $w_{\mathrm{p}}^{\mathrm{AWGNC}}(\mathbf{x})=w_{\mathrm{p}}^{\mathrm{BSC}}(\mathbf{x})=w_{\mathrm{p}}^{\mathrm{BEC}}(\mathbf{x})=w_{\mathrm{H}}(\mathbf{x})$. Let us briefly comment on the significance of the above pseudo-weights. When transmitting over an AWGNC, it can be shown that the squared Euclidean distance from the point $+\mathbf{1}$ in signal space, which corresponds to the codeword $\mathbf{0}$, to the plane $\left\{\boldsymbol{\lambda} \in \mathbb{R}^{n} \mid\langle\boldsymbol{\omega}, \boldsymbol{\lambda}\rangle=0\right\}$ is $w_{\mathrm{p}}^{\mathrm{AWGNC}}(\boldsymbol{\omega})$. When transmitting over a BSC, the LP decoder decides in favor of $\mathbf{0}$ and against $\boldsymbol{\omega}$ if the number of bit-flips on the BSC is smaller than $w_{\mathrm{p}}^{\mathrm{BSC}}(\boldsymbol{\omega}) / 2$; on the other hand, there is at least one pattern with at least $w_{\mathrm{p}}^{\mathrm{BSC}}(\boldsymbol{\omega}) / 2$ bit flips such that the LP decoder decides in favor of $\boldsymbol{\omega}$ and against $\mathbf{0}$, assuming that ties are resolved randomly. Finally, when transmitting over a BEC, the LP decoder decides in favor of $\mathbf{0}$ and against $\boldsymbol{\omega}$ if the number of erasures on the $\mathrm{BEC}$ is smaller than $w_{\mathrm{p}}^{\mathrm{BEC}}(\boldsymbol{\omega})$; on the other hand, there is a pattern with $w_{\mathrm{p}}^{\mathrm{BEC}}(\boldsymbol{\omega})$ erasures such that the LP decoder decides in favor of $\boldsymbol{\omega}$ and against $\mathbf{0}$ (assuming that ties are resolved randomly). For a more detailed discussion, see [4, 23. 
Definition 7 Let $\mathcal{C}$ be an arbitrary binary linear code. We recall the definition of the codeword weight enumerator

$$
\chi_{\mathcal{C}}^{\mathrm{cw}}(X) \triangleq \sum_{\mathbf{x} \in \mathcal{C}} X^{w_{\mathrm{H}}(\mathbf{x})}
$$

and define the minimal codeword weight enumerator, and the minimal pseudo-codeword AWGNC pseudo-weight enumerator, to be, respectively, the polynomials (with potentially non-integer exponents)

$$
\begin{aligned}
\chi_{\mathcal{C}}^{\mathrm{mcw}}(X) & \triangleq \sum_{\mathbf{x} \in \mathcal{M}(\mathcal{C})} X^{w_{\mathrm{H}}(\mathbf{x})} \\
\chi_{\mathbf{H}}^{\mathrm{mpcw}, \operatorname{AWGNC}}(X) & =\sum_{[\boldsymbol{\omega}] \in \mathcal{M}_{\mathrm{p}}(\mathcal{K}(\mathbf{H}))} X^{w_{\mathrm{p}}^{\mathrm{AWGNC}}(\boldsymbol{\omega})} .
\end{aligned}
$$

The summation in the last enumerator is over all equivalence classes of minimal pseudocodewords. ${ }^{9}$ (The minimal pseudo-codeword BSC pseudo-weight enumerator and the minimal pseudo-codeword BEC pseudo-weight enumerator are defined analogously.)

Instead of "weight enumerator" and "pseudo-weight enumerator" we will frequently use the words "weight spectrum" or "pseudo-weight spectrum", respectively, or simply "spectrum". Ideally, for a code defined by a parity-check matrix $\mathbf{H}$, we would like to know all the terms of the spectra that were defined in Def. [7. Often, we have to settle with less, in particular one often focuses on some quantities that characterize important aspects of a spectrum.

One such quantity is the minimum pseudo-weight: we let $w_{\mathrm{p}}^{\mathrm{AWGNC}, \min }(\mathbf{H}), w_{\mathrm{p}}^{\mathrm{BSC}, \min }(\mathbf{H})$, and $w_{\mathrm{p}}^{\mathrm{BEC}, \min }(\mathbf{H})$ be the minimum AWGNC, BSC, and BEC pseudo-weights of a paritycheck matrix $\mathbf{H}$, i.e. the minimum of the respective pseudo-weights, over all non-zero points in $\mathcal{K}(\mathbf{H})$. Using some simple tree-based techniques, or Th. 1 in [24, one can show that $w_{\mathrm{p}}^{\mathrm{AWGNC}, \min }\left(\mathbf{H}_{\mathrm{PG}(2, q)}\right) \geqslant q+2$ and because this lower bound matches the minimum Hamming weight, we actually know that $w_{\mathrm{p}}^{\mathrm{AWGNC}, \min }\left(\mathbf{H}_{\mathrm{PG}(2, q)}\right)=q+2$. Similarly, one can show that $w_{\mathrm{p}}^{\mathrm{BSC}, \min }\left(\mathbf{H}_{\mathrm{PG}(2, q)}\right)=w_{\mathrm{p}}^{\mathrm{BEC}, \min }\left(\mathbf{H}_{\mathrm{PG}(2, q)}\right)=q+2$ and that $w_{\mathrm{p}}^{\mathrm{AWGNC}, \min }\left(\mathbf{H}_{\mathrm{EG}(2, q)}\right)=w_{\mathrm{p}}^{\mathrm{BSC}, \min }\left(\mathbf{H}_{\mathrm{EG}(2, q)}\right)=w_{\mathrm{p}}^{\mathrm{BEC}, \min }\left(\mathbf{H}_{\mathrm{EG}(2, q)}\right)=q+1$.

Another important quantity that characterizes pseudo-weight spectra is the pseudoweight spectrum gap:

Definition 8 Let $\mathcal{C}$ be an arbitrary binary linear code described by the parity-check matrix $\mathbf{H}$ and let $\mathcal{M}_{\mathrm{p}}^{\prime}(\mathcal{K}(\mathbf{H})$ ) be the set of all minimal pseudo-codewords that are not multiples of minimal codewords. We call the real-valued quantity

$$
g_{\mathbf{H}}^{\mathrm{AWGNC}} \triangleq \min _{\boldsymbol{\omega} \in \mathcal{M}_{\mathrm{p}}^{\prime}(\mathcal{K}(\mathbf{H}))} w_{\mathrm{p}}^{\mathrm{AWGNC}}(\boldsymbol{\omega})-w_{\mathrm{H}}^{\min }(\mathcal{C}(\mathbf{H}))
$$

the $A W G N C$ pseudo-weight spectrum gap of $\mathbf{H}$. (The BSC pseudo-weight spectrum gap and the BEC pseudo-weight spectrum gap are defined analogously.)

\footnotetext{
${ }^{9}$ Two pseudo-codewords $\boldsymbol{\omega}, \boldsymbol{\omega}^{\prime} \in \mathcal{K}(\mathbf{H})$ are in the same equivalence class if there exists an $\alpha \in \mathbb{R}_{++}$such that $\boldsymbol{\omega}=\alpha \cdot \boldsymbol{\omega}^{\prime}$.
} 
Using [3, Cor. 8] (see also [4, Sec. 7]), one can show that for a randomly constructed $\left(w_{\text {col }}, w_{\text {row }}\right)$-regular code with $3 \leqslant w_{\text {col }}<w_{\text {row }}$ the AWGNC pseudo-weight spectrum gap becomes strictly negative with probability one as the block length goes to infinity. However, slightly extending the arguments that lead to the above mentioned minimum pseudo-weight results, one can show that for the $\mathrm{PG}(2, q)$ - and $\mathrm{EG}(2, q)$-based codes (with square paritycheck matrix as discussed in Sec. 10 the pseudo-weight spectrum gap is non-negative for finite $q$. In fact, we will see that for the codes investigated in Sec. 6 the pseudo-weight spectrum gap is significantly positive. We note that by applying simple performance bounding techniques it can be shown that the larger the gap is, the closer is the LP decoding performance (and potentially also the iterative decoding performance [3, 4]) to the ML decoding performance as the SNR goes to infinity. ${ }^{10}$

\section{Examples of Spectra}

In this section we exemplify many of the objects that were defined in the previous chapters, i.e. we present minimal pseudo-codewords, weight enumerators, and the pseudo-weight spectrum gap for some short PG(2,q)- and $\mathrm{EG}(2, q)$-based codes.

\subsection{Type-I PG-LDPC Code for $q=2$}

The PG(2,2)-based code $\mathcal{C}_{\mathrm{PG}(2,2)}$ has parameters $\left[n=7, k=3, d_{\min }=4\right]$ and can be represented by the following circulant parity-check matrix $\mathbf{H}_{\mathrm{PG}(2,2)}$ of size $7 \times 7$ :

$$
\mathbf{H}_{\mathrm{PG}(2,2)}=\left(\begin{array}{ccccccc}
1 & 1 & 0 & 1 & 0 & 0 & 0 \\
0 & 1 & 1 & 0 & 1 & 0 & 0 \\
0 & 0 & 1 & 1 & 0 & 1 & 0 \\
0 & 0 & 0 & 1 & 1 & 0 & 1 \\
1 & 0 & 0 & 0 & 1 & 1 & 0 \\
0 & 1 & 0 & 0 & 0 & 1 & 1 \\
1 & 0 & 1 & 0 & 0 & 0 & 1
\end{array}\right)
$$

It is not difficult to find out that the set $\mathcal{M}\left(\mathcal{C}_{\mathrm{PG}(2,2)}\right)$ of minimal codewords consists of the following codewords:

$$
\begin{aligned}
& (1,0,0,1,0,1,1), \\
& (1,1,0,0,1,0,1), \\
& (1,1,1,0,0,1,0), \\
& (0,1,1,1,0,0,1), \\
& (1,0,1,1,1,0,0), \\
& (0,1,0,1,1,1,0), \\
& (0,0,1,0,1,1,1) .
\end{aligned}
$$

Obviously, all of them have Hamming weight 4 and they are all cyclic shifts of each other. Because the code has $2^{3}=8$ codewords in total, it turns out that this code is special in the following sense: there are no non-zero codewords that are not minimal codewords.

\footnotetext{
${ }^{10}$ Of course, the pseudo-weight spectrum gap is only a first approximation to how quickly the LP decoding performance approaches the ML decoding performance as the SNR goes to infinity. A better approximation is given by initial parts (or the whole) minimal pseudo-codeword pseudo-weight enumerator.
} 
The set $\mathcal{M}_{\mathrm{p}}\left(\mathcal{K}\left(\mathbf{H}_{\mathrm{PG}(2,2)}\right)\right)$ of minimal codewords contains all the elements from $\mathcal{M}\left(\mathcal{C}_{\mathrm{PG}(2,2)}\right)$ plus the following pseudo-codewords that are not codewords (we show one representative per equivalence class):

$$
\begin{aligned}
& (1,2,2,1,2,1,1), \\
& (1,1,2,2,1,2,1), \\
& (1,1,1,2,2,1,2), \\
& (2,1,1,1,2,2,1), \\
& (1,2,1,1,1,2,2), \\
& (2,1,2,1,1,1,2), \\
& (2,2,1,2,1,1,1) .
\end{aligned}
$$

All these minimal pseudo-codewords that are not codewords are cyclic shifts of each other. The weight enumerators are given by:

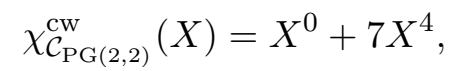

$$
\begin{aligned}
& \chi_{\mathcal{C}_{\mathrm{PG}(2,2)}^{\mathrm{mcw}}}(X)=7 X^{4},
\end{aligned}
$$

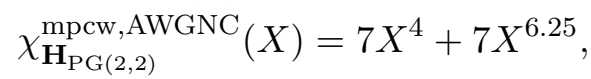

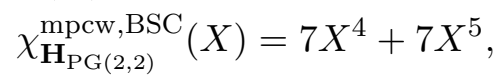

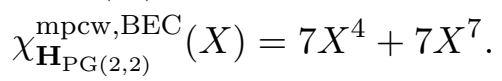

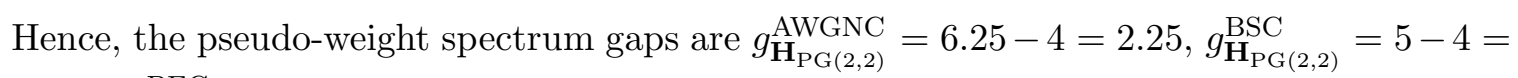
1 , and $g_{\mathbf{H}_{\mathrm{PG}(2,2)}^{\mathrm{BEC}}}^{\mathrm{BE}}=7-4=3$.

The codes introduced in Sec. 2 were based on square parity-check matrices. However, the code $\mathrm{PG}(2,2)$ can also be described by a parity-check matrix of size $4 \times 7$ such as

$$
\mathbf{H}_{\mathrm{PG}(2,2)}^{\prime}=\left(\begin{array}{ccccccc}
1 & 1 & 0 & 1 & 0 & 0 & 0 \\
0 & 1 & 1 & 0 & 1 & 0 & 0 \\
0 & 0 & 1 & 1 & 0 & 1 & 0 \\
0 & 0 & 0 & 1 & 1 & 0 & 1
\end{array}\right),
$$

which is the matrix formed by the first four lines of the $\mathbf{H}_{\mathrm{PG}(2,2)}$. Because $\mathbf{H}_{\mathrm{PG}(2,2)}^{\prime}$ contains a subset of the rows of $\mathbf{H}_{\mathrm{PG}(2,2)}$ it is clear that $\mathcal{K}\left(\mathbf{H}_{\mathrm{PG}(2,2)}\right) \subseteq \mathcal{K}\left(\mathbf{H}_{\mathrm{PG}(2,2)}^{\prime}\right)$. Moreover, note that a minimal pseudo-codeword of $\mathcal{K}\left(\mathbf{H}_{\mathrm{PG}(2,2)}\right)$ does not need to be minimal pseudocodeword of $\mathcal{K}\left(\mathbf{H}_{\mathrm{PG}(2,2)}^{\prime}\right)$. Indeed, the set of minimal pseudo-codewords that are not codewords turns out to be the union of the following sets (in which we show one representative per equivalence class):

$$
\begin{aligned}
& \{(3,2,1,1,1,0,0)\},\{(0,1,2,1,1,3,0)\},\{(0,1,1,1,2,0,3)\},\{(0,1,1,1,1,0,0)\}, \\
& \{(2,1,1,1,0,0,1),(1,2,1,1,1,0,0),(0,1,2,1,1,1,0)\}, \\
& \{(0,1,1,1,0,2,1),(1,0,1,1,1,0,2),(2,1,0,1,1,1,0)\}, \\
& \{(0,1,1,1,2,0,1)(1,0,1,1,1,2,0),(0,1,0,1,1,1,2)\},
\end{aligned}
$$

where cyclic shifts of the same pseudo-codeword are grouped in the same set. It is interesting to see that, for $\mathbf{H}_{\mathrm{PG}(2,2)}^{\prime}$, a cyclic shift of a minimal pseudo-codeword is not necessarily a (minimal) pseudo-codeword, as it was in the case of the matrix $\mathbf{H}_{\mathrm{PG}(2,2)}$. 
It follows that

$$
\begin{gathered}
\chi_{\mathbf{H}_{\mathrm{PG}(2,2)}^{\prime m p c w}, \mathrm{AWGNC}}^{\mathrm{mp}}(X)=11 X^{4}+9 X^{4.5}, \\
\chi_{\mathbf{H}_{\mathrm{PG}(2,2)}^{\mathrm{mpcw}, \mathrm{BSC}}}^{\mathrm{m}}(X)=3 X^{3}+17 X^{4}, \\
\chi_{\mathbf{H}_{\mathrm{PG}(2,2)}^{\prime}}^{\mathrm{mpcw}, \mathrm{BEC}}(X)=8 X^{4}+12 X^{5},
\end{gathered}
$$

and that the pseudo-weight spectrum gaps are $g_{\mathbf{H}_{\mathrm{PG}(2,2)}^{\prime}}^{\mathrm{AWGNC}}=4-4=0, g_{\mathbf{H}_{\mathrm{PG}(2,2)}^{\prime}}^{\mathrm{BSC}}=3-4=$

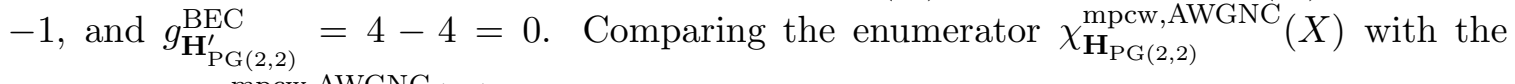

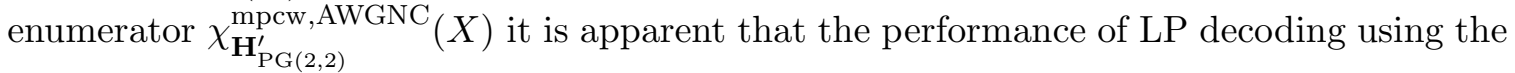
second representation will be worse than the performance of LP decoding using the first representation. Based on iterative decoder simulations, MacKay and Davey [25, Sec. 4] observed a similar performance hierarchy between different representations of the same code. Note that the code under investigation in [25] was the PG(2,16)-based code. Similar statements can be made for the BSC and the BEC.

Before concluding this subsection, let us comment on the vector $\boldsymbol{\omega} \triangleq(0,1,1,1,1,0,0)$, which is a minimal pseudo-codeword for $\mathbf{H}_{\mathrm{PG}(2,2)}^{\prime}$ but not a codeword for $\mathcal{C}_{\mathrm{PG}(2,2)}$, even though it has only 0 and 1 components. From our remarks after Lemma 4 , it follows that $\boldsymbol{\omega}$ cannot be an unscaled pseudo-codeword because $\boldsymbol{\omega}(\bmod 2)$ is not a codeword. However, its equivalent $(0,2,2,2,2,0,0)$ is an unscaled pseudo-codeword, and it stems from a triple cover.

Non-codeword pseudo-codewords that contain only zero and one components will be discussed again in Th. 13 .

\subsection{Type-I PG-LDPC Code for $q=4$}

The parity-check matrix $\mathbf{H}_{\mathrm{PG}(2,4)}$ of the $\mathrm{PG}(2,4)$-based code $\mathcal{C}_{\mathrm{PG}(2,4)}$ has size $21 \times 21$, uniform column and row weight 5 , and yields a code with parameters $\left[n=21, k=11, d_{\text {min }}=6\right]$. The codeword weight enumerator and the minimal codeword weight enumerator are

$$
\begin{aligned}
& \chi_{\mathcal{C}_{\mathrm{PG}(2,4)}^{\mathrm{cw}}}^{\mathrm{m}}(X)=X^{0}+168 X^{6}+210 X^{8}+1008 X^{10}+280 X^{12}+360 X^{14}+21 X^{16}, \\
& \chi_{\mathcal{C}_{\mathrm{PG}(2,4)}}^{\mathrm{mcw}}(X)=168 X^{6}+210 X^{8}+1008 X^{10},
\end{aligned}
$$

respectively. Looking at these enumerators we see that all codewords with Hamming weight 6,8 , and 10 are minimal codewords. Analyzing the set of all weight- 6 codewords one sees that they all have the same pattern, i.e. they can all be obtained from a single weight- 6 codeword by applying a suitable $\mathrm{PG}(2,4)$-automorphism. The same is true for all other sets of codewords with the same weight. This makes the classification of all the codewords of $\mathcal{C}_{\mathrm{PG}(2,4)}$, and in particular of the minimal codewords of $\mathcal{C}_{\mathrm{PG}(2,4)}$, relatively easy.

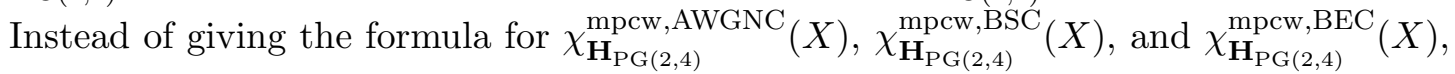
we simply show their histogram, cf. Fig. [1] Without going into any details, it is apparent from Fig. 1 that the influence of minimal pseudo-codewords can vary depending on the channel that is used. (For related observations about varying influences of minimal pseudo- 

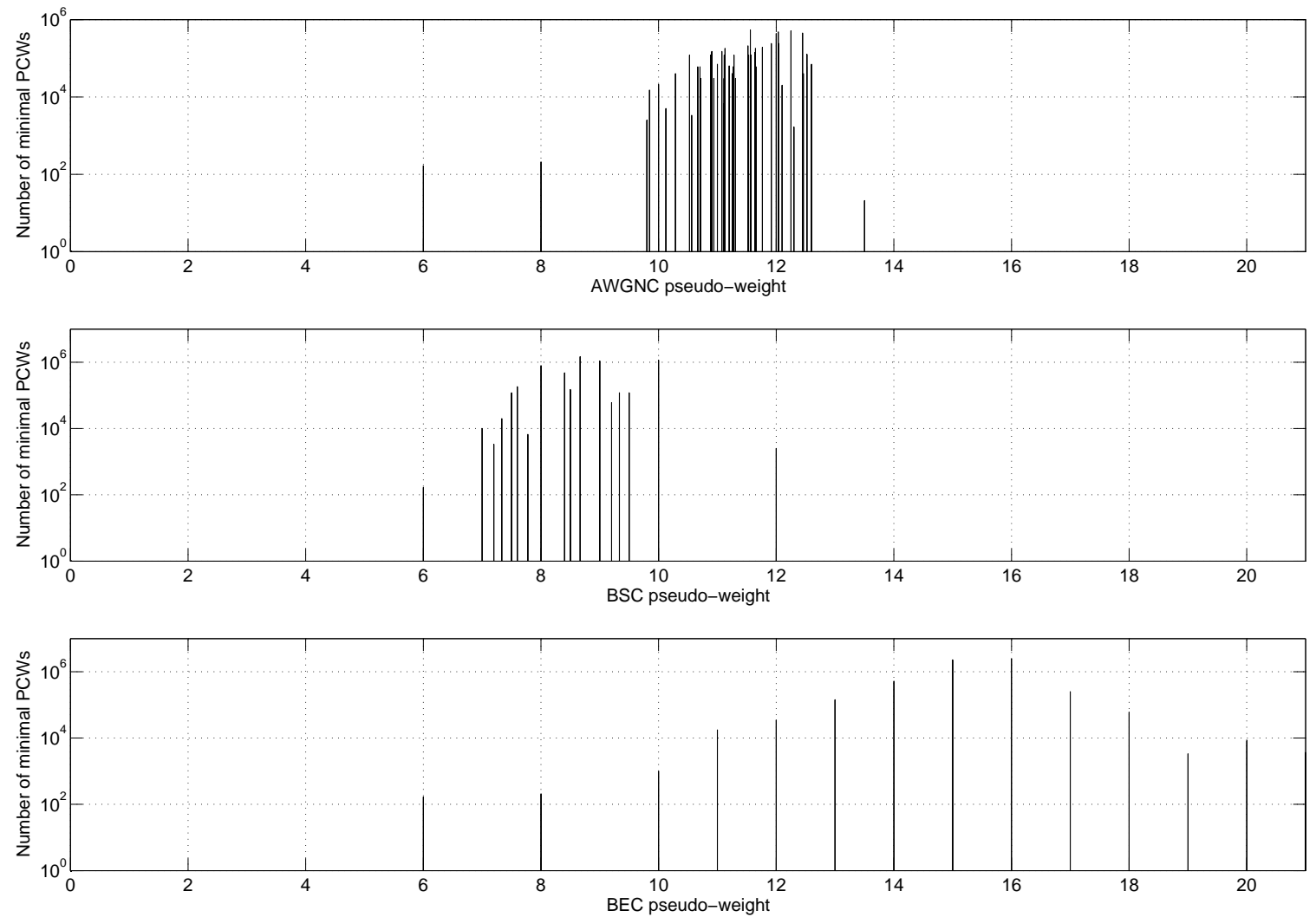

Figure 1: Histograms of the AWGNC, BSC, and BEC pseudo-weight of minimal pseudocodewords (PCWs) of the PG(2,4)-based code. (Note that the y-axes are logarithmic.) 
codewords, see also the discussion in [26].) The pseudo-weight gaps turn out to be

$$
\begin{aligned}
& g_{\mathbf{H}_{\mathrm{PG}(2,4)}}^{\mathrm{AWGNC}}=9.8-6=3.8, \\
& g_{\mathbf{H}_{\mathrm{PG}(2,4)}^{\mathrm{BSC}}}^{\mathrm{BC}}=7-6=1, \\
& g_{\mathbf{H}_{\mathrm{PG}(2,4)}}^{\mathrm{BEC}}=11-6=5 .
\end{aligned}
$$

We refer to the end of Sec. 5 for a discussion on the significance of positive pseudo-weight gaps.

\subsection{Type-I PG-LDPC Code for $q=8$}

The parity-check matrix $\mathbf{H}_{\mathrm{PG}(2,8)}$ of the $\mathrm{PG}(2,8)$-based code $\mathcal{C}_{\mathrm{PG}(2,8)}$ has size $73 \times 73$, uniform column and row weight 9 , and yields a code with parameters $\left[n=73, k=45, d_{\min }=10\right]$. Judging from some random search experiments in the fundamental cone $\mathcal{K}\left(\mathbf{H}_{\mathrm{PG}(2,8)}\right)$, the AWGNC pseudo-weight spectrum gap $g_{\mathbf{H}_{\mathrm{PG}(2,8)}}^{\mathrm{AWGNC}}$ seems to be at least 6.0.

\subsection{Type-I EG-LDPC Code for $q=4$}

The parity-check matrix $\mathbf{H}_{\mathrm{EG}(2,4)}$ of the $\mathrm{EG}(2,4)$-based code $\mathcal{C}_{\mathrm{EG}(2,4)}$ has size $15 \times 15$, uniform column and row weight 4 , and yields a code with parameters $\left[n=15, k=7, d_{\min }=5\right]$. The codeword weight enumerator and the minimal codeword weight enumerator are

$$
\begin{aligned}
& \chi_{\mathcal{C}_{\mathrm{EG}(2,4)}^{\mathrm{cw}}}^{\mathrm{m}}(X)=X^{0}+18 X^{5}+30 X^{6}+15 X^{7}+15 X^{8}+30 X^{9}+18 X^{10}+X^{15}, \\
& \chi_{\mathcal{C}_{\mathrm{EG}(2,4)}^{\mathrm{mcw}}}(X)=18 X^{5}+30 X^{6}+15 X^{7}+15 X^{8}+30 X^{9},
\end{aligned}
$$

respectively. Looking at these enumerators we see that all codewords with Hamming weight $5,6,7,8$, and 9 are minimal codewords. Analyzing the set of all weight-5 codewords one sees that they all have the same pattern, i.e. they can all be obtained from a single weight- 5 codeword by applying a suitable EG(2,4)-automorphism. The same is true for all other sets of codewords with the same weight.

The histograms in Fig. 2 correspond to various parity-check matrices that describe

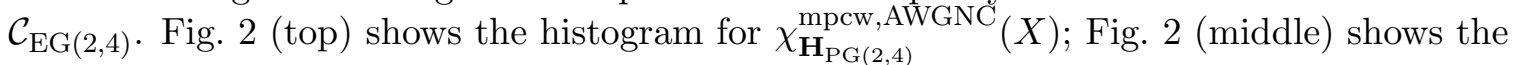
histogram for $\chi_{\mathbf{H}_{\mathrm{PG}(2,4)}^{\prime}}^{\mathrm{mpcw}, \mathrm{AWGNC}}(X)$ where $\mathbf{H}_{\mathrm{PG}(2,4)}^{\prime}$ is a randomly selected $9 \times 15$ submatrix (with column weights at least 2) of $\mathbf{H}_{\mathrm{PG}(2,4)}$; and finally Fig. 2 (bottom) shows the histogram for $\chi_{\mathbf{H}_{\mathrm{PG}(2,4)}^{\prime \prime}}^{\mathrm{mpcw}, \mathrm{AWGNC}}(X)$ where $\mathbf{H}_{\mathrm{PG}(2,4)}^{\prime \prime}$ is an $8 \times 15$ submatrix (with five columns having weight only one) of consecutive rows of the (circulant) matrix $\mathbf{H}_{\mathrm{PG}(2,4)}$. It can easily be seen that for the parity-check matrices under investigation those with more dependent rows lead to more favorable histograms.

In Fig. 3 we show various decoding simulation results for data transmission over a

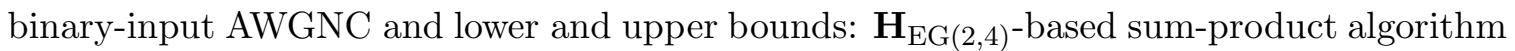

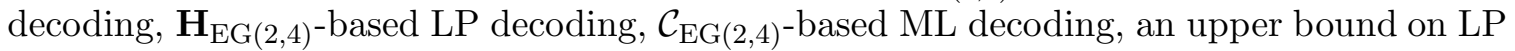
decoding based on a union of events upper bound, an upper bound on ML decoding based on a union of events upper bound, and a lower bound on ML decoding based on an inequality by de Caen as presented by Séguin [27]. It can be seen that thanks to the knowledge of minimal codewords and minimal pseudo-codewords we are able to obtain bounds that are very tight from a certain SNR value on. This is witnessed by the fast decreasing line labeled "LP UUB minus ML SLB" which shows the difference between the union upper bound on 

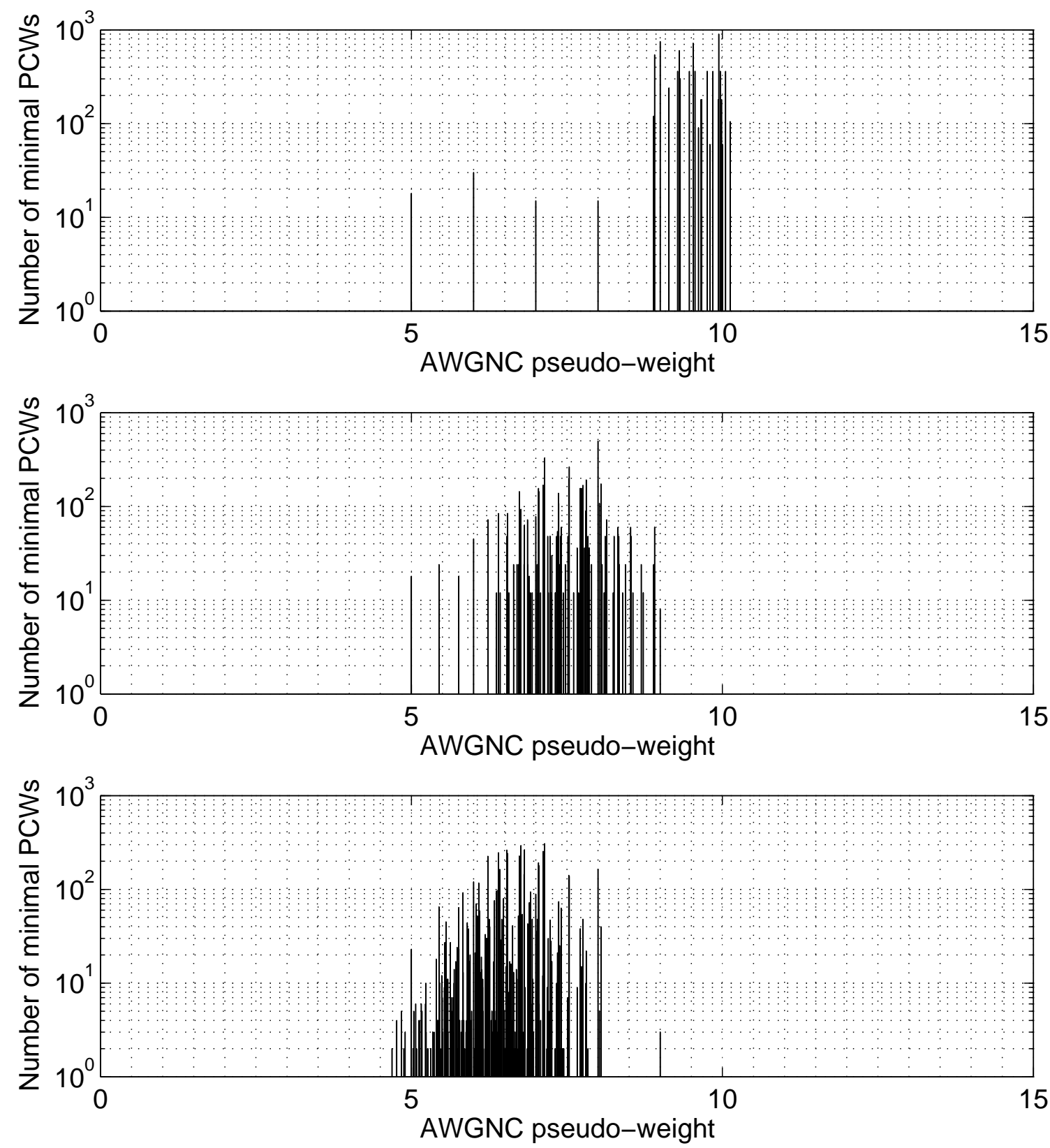

Figure 2: Histogram of the AWGNC pseudo-weight of minimal pseudo-codewords (PCWs) of the EG(2,4)-based code. (Note that the y-axes are logarithmic.) Top: For $15 \times 15$

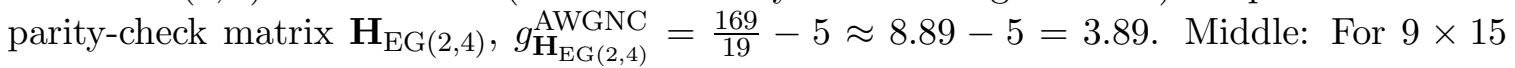

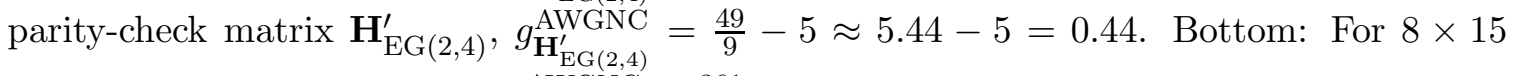
parity-check matrix $\mathbf{H}_{\mathrm{EG}(2,4)}^{\prime \prime}, g_{\mathbf{H}_{\mathrm{EG}(2,4)}^{\prime \prime}}^{\mathrm{AWGNC}}=\frac{361}{77}-5 \approx 4.69-5=-0.31$. 


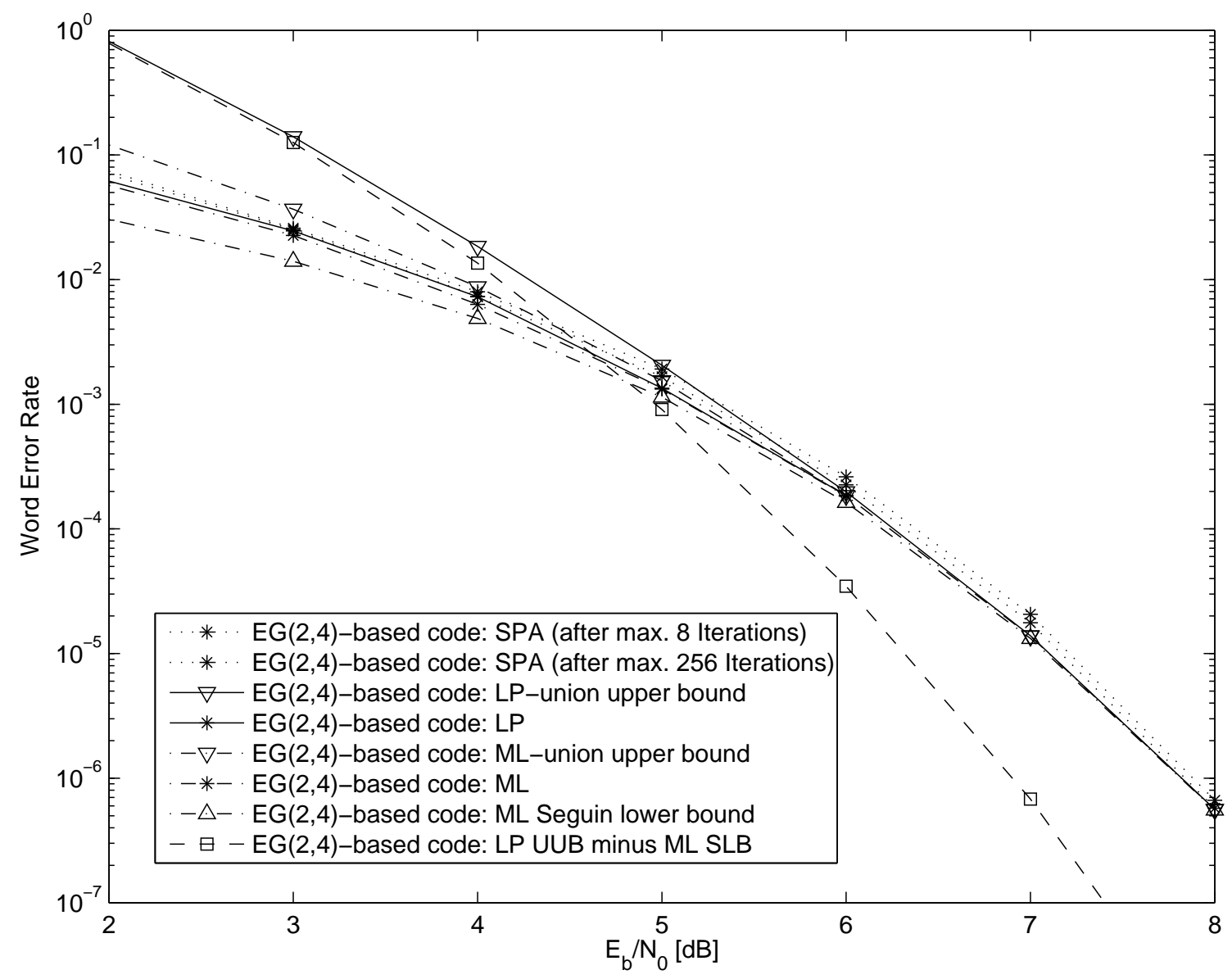

Figure 3: Word error rate for various decoding algorithms together with some upper and lower bounds. (See main text for explanations.) 
the LP decoder word error rate and the Séguin lower bound on the ML decoder word error rate.

\subsection{How the Results Were Obtained}

Let us briefly mention how the results for the minimal pseudo-codewords were obtained in Secs. 6.1 6.2 and 6.4 We used the program "lrs" 28] to search edges in cones. For the code $\mathcal{C}_{\mathrm{PG}(2,4)}$ in Sec. 6.2 we additionally used the two-transitivity of the points of a projective plane in order to formulate a simpler edge-enumeration subproblem which can be solved efficiently and from which all the minimal pseudo-codewords can be derived. This goes as follows: it is clear that a minimal pseudo-codeword cannot only fulfill inequalities of type (5) with equality (because only $\boldsymbol{\omega}=\mathbf{0}$ does this), i.e. at least one inequality of type (4) must be fulfilled with equality. Because the automorphism group of $\mathrm{PG}(2,4)$ is two-transitive (which means that for any two pair of points there exists an automorphism that maps the first pair to the second pair), we can pick any of the inequalities of type (5) to be fulfilled with equality. Solving the edge enumeration problem for this $n-1$ dimensional cone is simpler and using the automorphism group we can derive from this the minimal pseudo-codewords of the cone of interest.

\subsection{Connection to Stopping Sets}

Before finishing this section that showed some pseudo-weight enumerators, let us comment on stopping set weight enumerators for PG-based codes that were investigated by Kashyap and Vardy [29]. These stopping set weight enumerators are tightly related to the BEC pseudo-weight enumerators because of the following reasons [3, 4, 2]: the support of any pseudo-codeword is a stopping set whose size equals the BEC pseudo-weight of the pseudocodeword. Moreover, for every stopping set there is at least one pseudo-codeword whose support equals that stopping set. However, some care must be taken when comparing a BEC pseudo-weight enumerator and a stopping set weight enumerator because the former is a sum over minimal pseudo-codewords whereas the latter is a sum over minimal stopping sets. (See also the discussion in Sec. 8 on effective minimal pseudo-codewords for the BEC.)

\section{Bounds on the AWGNC Pseudo-Weight}

In this section we will give some bounds on the AWGNC pseudo-weight. First we discuss some general bounds on the pseudo-weight of arbitrary vectors in $\mathbb{R}_{+}^{n}$; note that these bounds will depend only on the type of the vector. Then we discuss some bounds for certain selected pseudo-codewords that appear in the fundamental cone of PG-based codes. For easier notation, if no confusion arises, we will use the shorter $w_{\mathrm{p}}(\boldsymbol{\omega})$ instead of $w_{\mathrm{p}}^{\mathrm{AWGNC}}(\boldsymbol{\omega})$.

Definition 9 Let $\boldsymbol{\omega} \in \mathbb{R}_{+}^{n}$ and let $t_{\ell} \triangleq t_{\ell}(\boldsymbol{\omega})$ be the number of components of the vector $\boldsymbol{\omega}$ that are equal to $\ell$, where $\ell \in \mathbb{R}_{+}$. Then, we call $\mathbf{t} \triangleq \mathbf{t}(\boldsymbol{\omega})=\left(t_{\ell}(\boldsymbol{\omega})\right)_{\ell \in \mathbb{R}_{+}}$the type of $\boldsymbol{\omega}$. (Note that in the following we do not assume that $\ell$ is a non-negative integer, only that it is a non-negative real number.)

It follows from this definition that only finitely many $t_{\ell}$ 's are non-zero and that $\sum_{\ell} t_{\ell}=$ $|\mathcal{I}|=n$ for any $\boldsymbol{\omega} \in \mathbb{R}_{+}^{n}$. Moreover, because $|\operatorname{supp}(\boldsymbol{\omega})|=\sum_{\ell>0} t_{\ell},\|\boldsymbol{\omega}\|_{1}=\sum_{\ell} \ell t_{\ell}$, and 
$\|\boldsymbol{\omega}\|_{2}^{2}=\sum_{\ell} \ell^{2} t_{\ell}$, we have

$$
w_{\mathrm{p}}^{\mathrm{AWGNC}}(\boldsymbol{\omega})=\frac{\left(\sum_{\ell} \ell t_{\ell}\right)^{2}}{\sum_{\ell} \ell^{2} t_{\ell}}, \text { and } w_{\mathrm{p}}^{\mathrm{BEC}}(\boldsymbol{\omega})=\sum_{\ell>0} t_{\ell} .
$$

If $\tilde{\boldsymbol{\omega}}=\alpha \cdot \boldsymbol{\omega}$ for some $\alpha \in \mathbb{R}_{++}$then its type $\tilde{\mathbf{t}} \triangleq \mathbf{t}(\tilde{\boldsymbol{\omega}})$ is such that $\tilde{t}_{\alpha \ell}=t_{\ell}$ for all $\ell$.

Lemma 10 Let $\boldsymbol{\omega} \in \mathbb{R}_{+}^{n}$ and let $\eta \neq 0$ be some arbitrary real number. Then

$$
w_{\mathrm{p}}(\boldsymbol{\omega}) \geqslant \frac{2 \eta\|\boldsymbol{\omega}\|_{1}-\|\boldsymbol{\omega}\|_{2}^{2}}{\eta^{2}}=\frac{\sum_{i=1}^{n} \omega_{i}\left(2 \eta-\omega_{i}\right)}{\eta^{2}}
$$

with equality if and only if $\boldsymbol{\omega}=\mathbf{0}$ or $\eta=\|\boldsymbol{\omega}\|_{2}^{2} /\|\boldsymbol{\omega}\|_{1}$.

Proof: If $\boldsymbol{\omega}=\mathbf{0}$ then the statement is certainly true, so let us assume that $\boldsymbol{\omega} \neq \mathbf{0}$. The square of any real number is non-negative, therefore $\left(\eta\|\boldsymbol{\omega}\|_{1}-\|\boldsymbol{\omega}\|_{2}^{2}\right)^{2} \geqslant 0$, with equality if and only if $\eta=\|\boldsymbol{\omega}\|_{2}^{2} /\|\boldsymbol{\omega}\|_{1}$, which, after rearranging, gives $\eta^{2}\|\boldsymbol{\omega}\|_{1}^{2} \geqslant 2 \eta\|\boldsymbol{\omega}\|_{1}\|\boldsymbol{\omega}\|_{2}^{2}-\|\boldsymbol{\omega}\|_{2}^{4}$. Finally, dividing by $\eta^{2}\|\boldsymbol{\omega}\|_{2}^{2}$ and using the definition of $w_{\mathrm{p}}(\boldsymbol{\omega})$, we obtain the desired result.

Corollary 11 Let $\boldsymbol{\omega} \in \mathbb{R}_{+}^{n}$, let $\mathbf{t} \triangleq \mathbf{t}(\boldsymbol{\omega})$ be the type of $\boldsymbol{\omega}$, and let $\eta \neq 0$ be some arbitrary real number. Then

$$
w_{\mathrm{p}}(\boldsymbol{\omega}) \geqslant \sum_{\ell} \beta_{\ell} t_{\ell} \quad \text { with } \quad \beta_{\ell}=\frac{\ell(2 \eta-\ell)}{\eta^{2}}=1-\left(1-\frac{\ell}{\eta}\right)^{2} .
$$

Proof: The result follows immediately from Lemma 10,

Corollary 12 Let $\boldsymbol{\omega} \in \mathbb{R}_{+}^{n}$ and let $\mathbf{t} \triangleq \mathbf{t}(\boldsymbol{\omega})$. Moreover, let $r$ be the ratio of the largest positive $\ell$ such that $t_{\ell}$ is non-zero and the smallest positive $\ell$ such that $t_{\ell}$ is non-zero. Then we have the lower bound

$$
w_{\mathrm{p}}(\boldsymbol{\omega}) \geqslant \frac{4 r}{(r+1)^{2}} \cdot|\operatorname{supp}(\boldsymbol{\omega})| .
$$

This bound was also obtained by Wauer [30] using a different derivation.

Proof: Let $m$ be the largest positive $\ell$ such that $t_{\ell}$ is non-zero and let $m^{\prime}$ be the smallest positive $\ell$ such that $t_{\ell}$ is non-zero. These definitions obviously yield $r=m / m^{\prime}$. Consider Cor. 11 with $\eta=\frac{m+m^{\prime}}{2}$. We obtain $w_{\mathrm{p}}(\boldsymbol{\omega}) \geqslant \sum_{\ell} \beta_{\ell} t_{\ell}$ (a) with $\beta_{\ell}=4 \ell \frac{m+m^{\prime}-\ell}{\left(m+m^{\prime}\right)^{2}}=1-(1-$ $\left.\frac{2 \ell}{m+m^{\prime}}\right)^{2}$. We observe that $\beta_{m^{\prime}}=\beta_{m}=\frac{4 m m^{\prime}}{\left(m+m^{\prime}\right)^{2}}=\frac{4 r}{(r+1)^{2}}$. Since $\beta_{\ell}$ is strictly concave in $\ell$ we must have $\beta_{\ell}>\beta_{m^{\prime}}=\beta_{m}=\frac{4 r}{(r+1)^{2}}$ for all $m^{\prime}<\ell<m$. It follows that

$$
w_{\mathrm{p}}(\boldsymbol{\omega})=\sum_{m^{\prime} \leqslant \ell \leqslant m} \beta_{\ell} t_{\ell} \geqslant \sum_{m^{\prime} \leqslant \ell \leqslant m} \frac{4 r}{(r+1)^{2}} t_{\ell}=\frac{4 r}{(r+1)^{2}} \sum_{m^{\prime} \leqslant \ell \leqslant m} t_{\ell}=\frac{4 r}{(r+1)^{2}} \cdot|\operatorname{supp}(\boldsymbol{\omega})| .
$$

Under the same assumptions as in Cor. 12, Kelley and Sridhara 31] proved that $w_{\mathrm{p}}(\boldsymbol{\omega}) \geqslant$ $\frac{2 r^{2}}{\left(1+r^{2}\right)(r-1)+2 r}|\operatorname{supp}(\boldsymbol{\omega})|$. Note that for $r=1$ and $r=2$ the bound in Cor. 12 equals this 
bound and that for integers $r$ larger than 2 the bound in Cor. 12 is larger than this bound. (Note that it follows from some simple considerations that for a minimal pseudo-codeword $\boldsymbol{\omega}$ the ratio $r$ is 1 or at least 2.)

In the following subsections we will give bounds on the AWGNC pseudo-weight of the class of minimal pseudo-codewords having the property that their components are all small non-negative integers. Based on the examples in Sec. 6] we formed the belief that for the Tanner graph families under study that the non-codeword minimal pseudo-codewords with small AWGNC pseudo-weight are from this class.

\subsection{Pseudo-Codewords with Zeros and Ones}

We start our analysis with pseudo-codewords of smallest possible entries, i.e. pseudocodewords with zeros and ones. The following theorem gives a lower bound on their weight.

Theorem 13 Let $\mathbf{H} \triangleq \mathbf{H}_{\mathrm{PG}(2, q)}$ and let $\boldsymbol{\omega} \in \mathcal{K}(\mathbf{H})$ be a (not necessarily minimal) pseudocodeword of type $\mathbf{t}$ with $t_{0}$ non-negative, $t_{1}$ positive, and $t_{\ell}=0$ otherwise. If $\boldsymbol{\omega}$ is not a codeword in $\mathcal{C}_{\mathrm{PG}(2, q)}$ then

$$
w_{\mathrm{p}}(\boldsymbol{\omega}) \geqslant\left\lceil\frac{q}{2}+1+\frac{1}{2} \sqrt{q^{2}+16 q+16}\right\rceil \geqslant q+4 .
$$

Proof: See Sec. A.1.

Note that the above bound yields, for $q=2$ and $q=4, w_{\mathrm{p}}(\boldsymbol{\omega}) \geqslant q+4$, and, for $q \geqslant 8$, $w_{\mathrm{p}}(\boldsymbol{\omega}) \geqslant q+5$, respectively.

Remark 14 An example of a non-codeword pseudo-codeword with only zeros and ones as discussed in Th. 13 was presented at the end of Sec. 6.1. Note, however, that this example was for $\mathbf{H}_{\mathrm{PG}(2,2)}^{\prime}$ in (7) and not for $\mathbf{H}_{\mathrm{PG}(2,2)}$ in (6).

Observations for small $\mathrm{PG}(2, q)$-based codes suggest the following conjecture.

Conjecture 15 Let $\mathbf{H} \triangleq \mathbf{H}_{\mathrm{PG}(2, q)}$ and let $\boldsymbol{\omega} \in \mathcal{K}(\mathbf{H})$ be a minimal pseudo-codeword of type $\mathbf{t}$ with both $t_{0}$ non-negative, $t_{1}$ positive, and $t_{\ell}=0$ otherwise. The vector $\boldsymbol{\omega}$ is then a minimal codeword.

The following theorem generalizes part 3 of Lemma 3 (regarding properties of minimal codewords) to pseudo-codewords with components equal only to zero and one.

Lemma 16 Let $\mathcal{C}$ be an $[n, k]$ binary linear code represented by a parity-check matrix $\mathbf{H}$. (Note that we do not assume that $\mathcal{C}=\mathcal{C}_{\mathrm{PG}(2, q)}$ or $\mathcal{C}=\mathcal{C}_{\mathrm{EG}(2, q)}$.) Let $\boldsymbol{\omega} \in \mathcal{K}(\mathbf{H})$ be a minimal pseudo-codeword of type $\mathbf{t}$ with $t_{0}$ non-negative, $t_{1}$ positive, and $t_{\ell}=0$ otherwise. Then

$$
w_{\mathrm{p}}(\boldsymbol{\omega})=w_{\mathrm{H}}(\boldsymbol{\omega}) \leqslant n-k+1 .
$$

Proof: Without loss of generality we can assume that the pseudo-codeword indices have been reordered such that the first $n_{1} \triangleq w_{\mathrm{H}}(\boldsymbol{\omega})$ components of $\boldsymbol{\omega}$ are equal to one and such that the remaining $n_{2} \triangleq n-w_{\mathrm{H}}(\boldsymbol{\omega})$ components of $\boldsymbol{\omega}$ are equal to zero.

Let $\mathbf{K}_{1} \boldsymbol{\omega} \geqslant \mathbf{0}$ be the collection of inequalities of type (4) that a pseudo-codeword must fulfill and let $\mathbf{K}_{2} \boldsymbol{\omega} \geqslant \mathbf{0}$ be the collection of inequalities of type (5) that a pseudo-codeword must fulfill. Then there exists a full-rank $\left(n_{1}-1\right) \times n$-submatrix $\mathbf{A}_{1} \triangleq\left(\mathbf{A}_{11} \mid \mathbf{A}_{12}\right)$ of 
$\mathbf{K}_{1}$ and a full-rank $n_{2} \times n$-submatrix of $\mathbf{A}_{2} \triangleq\left(\mathbf{A}_{21} \mid \mathbf{A}_{22}\right)$ of $\mathbf{K}_{2}$ such that $\mathbf{A}_{1} \boldsymbol{\omega}=\mathbf{0}$ and $\mathbf{A}_{2} \boldsymbol{\omega}=\mathbf{0}$, such that $\mathbf{A}_{21}=\mathbf{0}$ and $\mathbf{A}_{22}=\mathbf{I}_{n_{2}}$, and such that $\operatorname{rank}_{\mathbb{R}}(\mathbf{A})=n-1$, where $\mathbf{A} \triangleq\left(\begin{array}{l}\mathbf{A}_{1} \\ \mathbf{A}_{2}\end{array}\right)=\left(\begin{array}{ll}\mathbf{A}_{11} & \mathbf{A}_{12} \\ \mathbf{A}_{21} & \mathbf{A}_{22}\end{array}\right)=\left(\begin{array}{cc}\mathbf{A}_{11} & \mathbf{A}_{12} \\ \mathbf{0} & \mathbf{I}_{n_{2}}\end{array}\right)$. Applying elementary row operations to the matrix $\mathbf{A}$ we obtain the matrix $\tilde{\mathbf{A}}=\left(\begin{array}{cc}\mathbf{A}_{11} & \mathbf{0} \\ \mathbf{0} & \mathbf{I}_{n_{2}}\end{array}\right)$. Because all non-zero entries of $\boldsymbol{\omega}$ are equal to one and because $\boldsymbol{\omega}$ is assumed to be a minimal pseudo-codeword, it turns out that all rows of $\mathbf{A}_{11}$ must contain exactly two non-zero elements, one equal to +1 and one equal to -1 .

These facts can be used as follows. First, we will show that $\operatorname{rank}_{\mathbb{F}_{2}}\left(\mathbf{A}_{11}\right)=n_{1}-1$. Secondly, we will show that $\operatorname{rank}_{\mathbb{F}_{2}}\left(\mathbf{A}_{11}\right) \leqslant n-k$. Finally, combining these results we will obtain the desired statement that $w_{\mathrm{p}}(\boldsymbol{\omega})=w_{\mathrm{H}}(\boldsymbol{\omega})=n_{1} \leqslant n-k+1$.

So, let us show that $\operatorname{rank}_{\mathbb{F}_{2}}\left(\mathbf{A}_{11}\right)=n_{1}-1$, i.e. that $\operatorname{rank}_{\mathbb{F}_{2}}\left(\mathbf{A}_{11}\right)=\operatorname{rank}_{\mathbb{R}}\left(\mathbf{A}_{11}\right)$. Indeed, using the the special row structure of $\mathbf{A}_{11}$, it can be verified that the only vector in the (right-hand side) kernel of $\mathbf{A}_{11}(\bmod 2)$ is the all-ones vector over $\mathbb{F}_{2}$ of length $n_{1}$.

Secondly, let us show that $\operatorname{rank}_{\mathbb{F}_{2}}\left(\mathbf{A}_{11}\right) \leqslant n-k$. Indeed, we observe that every row in $\mathbf{A}_{1}(\bmod 2)$ corresponds to a row in $\mathbf{H}$. This implies that $\operatorname{rank}_{\mathbb{F}_{2}}\left(\mathbf{A}_{11}\right) \leqslant \operatorname{rank}_{\mathbb{F}_{2}}\left(\mathbf{A}_{1}\right) \leqslant$ $\operatorname{rank}_{\mathbb{F}_{2}}(\mathbf{H}) \leqslant n-k$.

Remark 17 A crucial element in the above proof was the fact that all rows of $\mathbf{A}_{11}$ contain exactly two non-zero entries, one equal to +1 and one equal to -1 . For minimal pseudocodewords where not all non-zero entries are equal, this is not the case anymore and therefore we cannot use the above proof to generalize the lemma statement to other types of minimal pseudo-codewords.

\subsection{Pseudo-Codewords with Zeros, Ones, and Twos}

Theorem 18 Let $\mathbf{H} \triangleq \mathbf{H}_{\mathrm{PG}(2, q)}$ and let $\boldsymbol{\omega} \in \mathcal{K}(\mathbf{H})$ be of type $\mathbf{t}$ with both $t_{0}$ non-negative, $t_{1}$ positive, $t_{2}$ positive, and $t_{\ell}=0$ otherwise. Then

$$
w_{\mathrm{p}}(\boldsymbol{\omega}) \geqslant \frac{32}{27}(q+2) \approx 1.185(q+2) .
$$

Proof: For any $i \in \mathcal{I}$ we must have $\sum_{i^{\prime} \in \mathcal{I} \backslash\{i\}} \omega_{i^{\prime}} \stackrel{(\text { a) }}{=} \sum_{j \in \mathcal{J}_{i}} \sum_{i^{\prime} \in \mathcal{I}_{j} \backslash\{i\}} \omega_{i^{\prime}} \stackrel{(\mathrm{b})}{\geqslant} \sum_{j \in \mathcal{J}_{i}} \omega_{i}=$ $(q+1) \omega_{i}$, where at step (a) we used the fact that all variable nodes are at graph distance two from each other in the Tanner graph associated to $\mathbf{H}$, and where at step (b) we used the inequalities in (4). Adding $\omega_{i}$ to both sides we obtain $\sum_{i^{\prime} \in \mathcal{I}} \omega_{i^{\prime}} \geqslant(q+2) \omega_{i}$. Now, fix an $i \in \mathcal{I}$ for which $\omega_{i}=2$ holds and express $\sum_{i^{\prime} \in \mathcal{I}} \omega_{i^{\prime}}$ in terms of $\mathbf{t}$ : it must hold that $t_{1}+2 t_{2} \geqslant 2(q+2)(\mathrm{c})$.

In a second step, we construct a vector $\boldsymbol{\omega}^{\prime}=\left(\omega_{1}^{\prime}, \ldots, \omega_{n}^{\prime}\right) \in \mathbb{R}^{n}$ such that

$$
\omega_{i}^{\prime} \triangleq\left\{\begin{array}{ll}
0 & \text { if } \omega_{i}=0 \\
2 & \text { if } \omega_{i}=1 \\
1 & \text { if } \omega_{i}=2
\end{array} \quad \text { for all } i \in \mathcal{I}\right)
$$

It can easily be seen that $\boldsymbol{\omega}^{\prime}$ lies also in the fundamental cone, i.e. $\boldsymbol{\omega}^{\prime} \in \mathcal{K}(\mathbf{H})$, and that $\boldsymbol{\omega}^{\prime}$ has type $\mathbf{t}^{\prime}$ with $t_{1}^{\prime}=t_{2}$ positive, $t_{2}^{\prime}=t_{1}$ positive, and $t_{\ell}^{\prime}=t_{\ell}$ otherwise. In other words, switching $0 \mapsto 0,1 \mapsto 2,2 \mapsto 1$ we obtain another pseudo-codeword. Arguing as above, for any $i \in \mathcal{I}$ we must have $\sum_{i^{\prime} \in \mathcal{I}} \omega_{i^{\prime}}^{\prime} \geqslant(q+2) \omega_{i}^{\prime}$. Now, fix an $i \in \mathcal{I}$ for which $\omega_{i}^{\prime}=2$ holds, and express $\sum_{i^{\prime} \in \mathcal{I}} \omega_{i^{\prime}}$ in terms of $\mathbf{t}^{\prime}$ : it must hold that $t_{1}^{\prime}+2 t_{2}^{\prime} \geqslant 2(q+2)$, i.e. that $t_{2}+2 t_{1} \geqslant 2(q+2)(\mathrm{d})$. 
Combining (c) and (d) we obtain $3\left(t_{1}+t_{2}\right) \geqslant 4(q+2)$, i.e. $|\operatorname{supp}(\boldsymbol{\omega})|=t_{1}+t_{2} \geqslant \frac{4}{3}(q+2)$. Using Cor. 12 we can conclude that $w_{\mathrm{p}}(\boldsymbol{\omega}) \geqslant \frac{4 \cdot 2}{(2+1)^{2}} \cdot \frac{4}{3}(q+2)=\frac{8}{9} \cdot \frac{4}{3}(q+2)=\frac{32}{27}(q+2)$.

Using some stronger assumptions on the pseudo-codeword $\boldsymbol{\omega}$ we can obtain a stronger lower bound, as is shown in the next theorem.

Theorem 19 Let $\mathbf{H} \triangleq \mathbf{H}_{\mathrm{PG}(2, q)}$ and let $\boldsymbol{\omega} \in \mathcal{K}(\mathbf{H})$ be of type $\mathbf{t}$ with both $t_{0}$ non-negative, $t_{1} \geqslant q+2, t_{2}$ positive, and $t_{\ell}=0$ otherwise. ${ }^{11}$ Then

$$
w_{\mathrm{p}}(\boldsymbol{\omega}) \geqslant \frac{4}{3}(q+2) \approx 1.333(q+2) .
$$

Proof: The start is similar to the beginning of the proof of Th. 18. For any $i \in \mathcal{I}$ we must have $\sum_{i^{\prime} \in \mathcal{I}} \omega_{i^{\prime}} \geqslant(q+2) \omega_{i}$. Now, fix an $i \in \mathcal{I}$ for which $\omega_{i}=2$ holds, and express $\sum_{i^{\prime} \in \mathcal{I}} \omega_{i^{\prime}}$ in terms of $\mathbf{t}$ : it must hold that $t_{1}+2 t_{2} \geqslant 2(q+2)$, or, equivalently, $t_{2} \geqslant q+2-t_{1} / 2$. For any $\eta \neq 0$ we obtain

$$
\begin{aligned}
w_{\mathrm{p}}(\boldsymbol{\omega}) & \stackrel{(\mathrm{a})}{\geqslant} \frac{(2 \eta-1) t_{1}+(4 \eta-4) t_{2}}{\eta^{2}} \stackrel{(\mathrm{b})}{\geqslant} \frac{(2 \eta-1) t_{1}+(4 \eta-4)\left(q+2-t_{1} / 2\right)}{\eta^{2}} \\
& =\frac{t_{1}+(4 \eta-4)(q+2)}{\eta^{2}},
\end{aligned}
$$

where at step (a) we used Cor. 11 and at step (b) we used the inequality on $t_{2}$ that we just found above. Using the assumption that $t_{1} \geqslant q+2$ from the theorem statement we get $w_{\mathrm{p}}(\boldsymbol{\omega}) \geqslant \frac{(4 \eta-3)(q+2)}{\eta^{2}}$. The right-hand side of this expression is maximized by $\eta^{*}=\frac{3}{2}$ : inserting this value yields the lower bound in the theorem statement.

Remark 20 Let $\mathcal{C}$ be the code defined by $\mathbf{H}$. If a pseudo-codeword is an unscaled pseudocodeword [20, 4] then it is equal (modulo 2) to a codeword of $\mathcal{C}$. Therefore, the number of odd components of an unscaled pseudo-codeword must either be zero or at least equal to the minimum Hamming weight of the code. So, if we actually know that $\boldsymbol{\omega}$ in Th. 19] is an unscaled pseudo-codeword then the requirement $t_{1} \geqslant q+2$ in the theorem statement is equivalent to the requirement $t_{1} \geqslant 1$.

Note also that Th. 19 can be generalized to the setup where $\boldsymbol{\omega} \in \mathcal{K}(\mathbf{H})$ has type $\mathbf{t}$ with $t_{0}$ non-negative, $t_{m}$ positive for some integer $m \geqslant 2$, $t_{\ell}$ non-negative for $1 \leqslant \ell \leqslant m-1$, $t_{\ell}=0$ for $\ell \geqslant m+1$, and $\sum_{\text {odd } \ell} t_{\ell} \geqslant q+2$. Then $w_{\mathrm{p}}(\boldsymbol{\omega}) \geqslant \frac{m^{2}}{m^{2}-m+1}(q+2)$.

Example 21 Let us exhibit some low-weight minimal pseudo-codewords that contain only zeros, ones, and twos. Consider first the case $q=2$. The projective plane for $q=2$ is shown in Fig. 4 (a): it has 7 points and 7 lines and we consider the points to be variables and the lines to be checks. Fig. 4 ( $\mathrm{a}$ and $\mathrm{b}$ ) shows two codewords of weight $q+2=4$; note that their supports overlap in $\frac{q+2}{2}=2$ positions. Adding these two codewords together yields the pseudo-codeword shown in Fig. 4 (c). Switching the zero value into a two results in the pseudo-codeword in Fig. 4 (d); it can be checked that this pseudo-codeword is actually a

\footnotetext{
${ }^{11}$ See Rem. 20 for a comment on these conditions.
} 


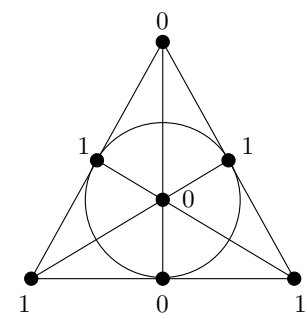

(a)

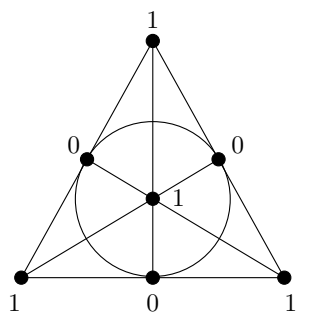

(b)

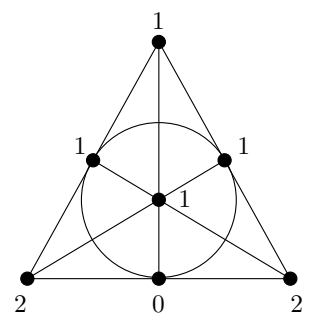

(c)

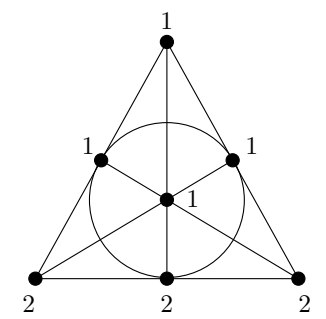

(d)

Figure 4: Codewords and pseudo-codewords used in Ex. 21]

minimal pseudo-codeword. It has AWGNC pseudo-weight 6.25, whereas the lower bounds in Ths. 18 and 19] are 4.74 and 5.33, respectively.

Similarly, in the case of $q=4$ it is possible to start with two codewords of weight $q+2=6$ whose supports overlap in $\frac{q+2}{2}=3$ positions. After adding them and switching two zeros (that are specifically chosen and lie on the same line) into two twos, one gets a minimal pseudo-codeword of AWGNC pseudo-weight 9.85, whereas the lower bounds in Ths. 18 and 19 are 7.11 and 8.00, respectively.

In the case $q=8$ it is possible to start with two codewords of weight $q+2=10$ whose supports overlap in $\frac{q+2}{2}=5$ positions. After adding them and switching three zeros (that are specifically chosen and form a triangle) into two twos, one gets a minimal pseudocodeword of AWGNC pseudo-weight 16.10, whereas the lower bound in Ths. 18 and [19] are 11.85 and 13.33 , respectively.

We conjecture that, with suitable generalizations, the above construction can be extended to larger $q$.

\subsection{Pseudo-Codewords with Zeros, Ones, Twos, and Threes}

Theorem 22 Let $\mathbf{H} \triangleq \mathbf{H}_{\mathrm{PG}(2, q)}$ and let $\boldsymbol{\omega} \in \mathcal{K}(\mathbf{H})$ be of type $\mathbf{t}$ with both $t_{0}$ non-negative, $t_{1}$ positive, $t_{2}$ non-negative, $t_{3}$ positive, and $t_{\ell}=0$ otherwise. We require that $\boldsymbol{\omega}$ is an unscaled pseudo-codeword. Then

$$
w_{\mathrm{p}}(\boldsymbol{\omega}) \geqslant \frac{9}{8} \cdot(q+2)=1.125(q+2)
$$

Proof: The start is similar to the beginning of the proof of Th. 18. For any $i \in \mathcal{I}$ we must have $\sum_{i^{\prime} \in \mathcal{I}} \omega_{i^{\prime}} \geqslant(q+2) \omega_{i}$. Now, fix an $i \in \mathcal{I}$ for which $\omega_{i}=3$ holds and express $\sum_{i^{\prime} \in \mathcal{I}} \omega_{i^{\prime}}$ in terms of $\mathbf{t}$ : it must hold that $t_{1}+2 t_{2}+3 t_{3} \geqslant 3(q+2)$ (a).

In a second step, we construct a vector $\boldsymbol{\omega}^{\prime}=\left(\omega_{1}^{\prime}, \ldots, \omega_{n}^{\prime}\right) \in \mathbb{R}^{n}$ such that

$$
\omega_{i}^{\prime} \triangleq\left\{\begin{array}{ll}
3 & \text { if } \omega_{i}=1 \\
2 & \text { if } \omega_{i}=2 \\
1 & \text { if } \omega_{i}=3 \\
0 & \text { otherwise }
\end{array} \quad \text { for all } i \in \mathcal{I}\right)
$$

It can be seen that $\boldsymbol{\omega}^{\prime}$ lies also in the fundamental cone, i.e. $\boldsymbol{\omega}^{\prime} \in \mathcal{K}(\mathbf{H}),{ }^{12}$ and that $\boldsymbol{\omega}^{\prime}$ has type $\mathbf{t}^{\prime}$ with $t_{1}^{\prime}=t_{3}$ positive, $t_{2}^{\prime}=t_{2}$ non-negative, $t_{3}^{\prime}=t_{1}$ positive, and $t_{\ell}^{\prime}=t_{\ell}$

\footnotetext{
${ }^{12}$ Note that the inequality $3+3 \geqslant 1$ goes into the inequality $1+1 \geqslant 3$, which is wrong. However, we assumed that $\boldsymbol{\omega}$ is an unscaled pseudo-codeword, which, among other things, implies that the modulo- 2 sum
} 
otherwise. Arguing as above, for any $i \in \mathcal{I}$ we must have $\sum_{i^{\prime} \in \mathcal{I}} \omega_{i^{\prime}}^{\prime} \geqslant(q+2) \omega_{i}^{\prime}$. Now, fix an $i \in \mathcal{I}$ for which $\omega_{i}^{\prime}=3$ holds and express $\sum_{i^{\prime} \in \mathcal{I}} \omega_{i^{\prime}}$ in terms of $\mathbf{t}^{\prime}$ : it must hold that $t_{1}^{\prime}+2 t_{2}^{\prime}+3 t_{3}^{\prime} \geqslant 3(q+2)$, i.e. that $t_{3}+2 t_{2}+3 t_{1} \geqslant 3(q+2)(\mathrm{b})$.

Combining (a) and (b) we obtain $4\left(t_{1}+t_{2}+t_{3}\right) \geqslant 6(q+2)$, i.e. $|\operatorname{supp}(\boldsymbol{\omega})|=t_{1}+t_{2}+t_{3} \geqslant$ $\frac{3}{2}(q+2)$. Using Cor. 12] we can conclude that $w_{\mathrm{p}}(\boldsymbol{\omega}) \geqslant \frac{4 \cdot 3}{(3+1)^{2}} \cdot \frac{3}{2}(q+2)=\frac{3}{4} \cdot \frac{3}{2}(q+2)=\frac{9}{8}(q+2)$.

\section{Effective Minimal Pseudo-Codewords}

The BSC can be seen as a binary-input AWGNC where the values at the output are quantized to +1 or -1 . It follows that the components of the log-likelihood vector $\boldsymbol{\lambda}$ can take on only two values, namely $+L$ and $-L$, where $L$ is a positive constant that depends on the bit flipping probability of the BSC. Because of this quantization, there are certain things that happen for the BSC that cannot happen for the AWGNC. Similarly, there are things that happen for the BEC that cannot happen for the AWGNC or the BSC. By introducing the so-called effectiveness of minimal pseudo-codewords, this section discusses some of these special behaviors.

Definition 23 Fix a memoryless binary-input channel and let $\mathcal{L}^{(n)} \subseteq(\mathbb{R} \cup\{ \pm \infty\})^{n}$ be the set of all possible log-likelihood ratio vectors upon sending the all-zero codeword. ${ }^{13}$ Moreover, let us fix a parity-check matrix $\mathbf{H}$ and let $\mathcal{M}_{\mathrm{p}}(\mathcal{K}(\mathbf{H}))$ be the set of minimal pseudo-codewords. A minimal pseudo-codeword $\boldsymbol{\omega} \in \mathcal{M}_{\mathrm{p}}(\mathcal{K}(\mathbf{H}))$ is called effective of the first kind for that particular channel if there exists a $\boldsymbol{\lambda} \in \mathcal{L}^{(n)}$ such that $\langle\boldsymbol{\omega}, \boldsymbol{\lambda}\rangle\left\langle 0\right.$ and $\left\langle\boldsymbol{\omega}^{\prime}, \boldsymbol{\lambda}\right\rangle \geqslant 0$ for all $\boldsymbol{\omega}^{\prime} \in \mathcal{M}_{\mathrm{p}}(\mathcal{K}(\mathbf{H})) \backslash\{\boldsymbol{\omega}\}$. A minimal pseudo-codeword $\boldsymbol{\omega} \in \mathcal{M}_{\mathrm{p}}(\mathcal{K}(\mathbf{H}))$ is called effective of the second kind for that particular channel if there exists a $\boldsymbol{\lambda} \in \mathcal{L}^{(n)}$ such that $\langle\boldsymbol{\omega}, \boldsymbol{\lambda}\rangle \leqslant 0$ and $\left\langle\boldsymbol{\omega}^{\prime}, \boldsymbol{\lambda}\right\rangle \geqslant 0$ for all $\boldsymbol{\omega}^{\prime} \in \mathcal{M}_{\mathrm{p}}(\mathcal{K}(\mathbf{H})) \backslash\{\boldsymbol{\omega}\}$. (Obviously, a minimal pseudo-codeword that is effective of the first kind is also effective of the second kind.)

Let $\mathcal{L}_{\mathbf{0}}^{(n)} \subseteq \mathcal{L}^{(n)}$ be the set where LP decoding decides in favor of the codeword $\mathbf{0}$. From the above definition it follows that a minimal pseudo-codeword "shapes" the set $\mathcal{L}_{\mathbf{0}}^{(n)}$ if and only if it is an effective minimal pseudo-codeword. More precisely, in the case where a minimal pseudo-codeword $\boldsymbol{\omega}$ is effective of the first kind then there exists at least one $\boldsymbol{\lambda} \in \mathcal{L}^{(n)}$ where $\boldsymbol{\omega}$ wins against all other minimal pseudo-codewords (and the zero codeword). Moreover, in the case where $\boldsymbol{\omega}$ is effective of the second kind we are guaranteed that there is at least one $\boldsymbol{\lambda} \in \mathcal{L}^{(n)}$ were $\boldsymbol{\omega}$ is involved in a tie; if and how often $\boldsymbol{\omega}$ wins against all other minimal pseudo-codewords (and the zero codeword) depends on how ties are resolved. ${ }^{14}$

of the $\omega_{i}$ 's that are involved in a check is zero. Therefore, it cannot happen that the non-zero $\omega_{i}$ 's that are involved in a check have the values 3,3 , and 1 .

${ }^{13}$ For the AWGNC we have $\mathcal{L}_{\mathrm{AWGNC}}^{(n)}=\mathbb{R}^{n}$, for the BSC we have $\mathcal{L}_{\mathrm{BSC}}^{(n)}=\{ \pm L\}^{n}$ for some $L \in \mathbb{R}_{++}$, and for the BEC we have $\mathcal{L}_{\mathrm{BEC}}^{(n)}=\{0,+\infty\}^{n}$. Please note that there was a slight mistake in [32, Def. 10], i.e. in [32, Def. 10] we forgot to require that the all-zeros codeword was sent. Nevertheless, compared to 32 Def. 10], the sets $\mathcal{L}_{\mathrm{AWGNC}}^{(n)}$ and $\mathcal{L}_{\mathrm{BSC}}^{(n)}$ remain unchanged, whereas for the BEC we have $\{0,+\infty\}^{n}$ instead of $\{-\infty, 0,+\infty\}^{n}$. Moreover, we assume that $L \in \mathbb{R}_{++}$and not $L \in \mathbb{R}_{+}$in order to avoid the uninteresting case $L=0$ stemming from a BSC with cross-over probability $1 / 2$.

${ }^{14}$ The fact that a minimal pseudo-codeword is effective of the second kind does of course not exclude the possibility that there are also $\lambda \in \mathcal{L}^{(n)}$ were $\boldsymbol{\omega}$ wins (unconditionally) against all other minimal pseudocodewords (and the zero codeword). 
Theorem 24 For the binary-input $A W G N C$ and any parity-check matrix $\mathbf{H}$ all minimal pseudo-codewords of $\mathcal{K}(\mathbf{H})$ are effective of the first kind.

Proof: This follows from basic cone properties (cf. e.g. 21]).

We now turn to the BSC. As the following observations show, for this channel not all minimal pseudo-codewords need to be effective of the first or of the second kind.

Theorem 25 Consider data transmission over a BSC using the code defined by $\mathbf{H} \triangleq$ $\mathbf{H}_{\mathrm{PG}(2, q)}$. Then LP decoding can correct any pattern of $\frac{q}{2}$ bit flips and no pattern of more than $q$ bit flips.

Proof: Because $w_{\mathrm{p}}^{\mathrm{BSC}, \min }(\mathbf{H})=q+2$, the BSC pseudo-weight of any pseudo-codeword in $\mathcal{K}(\mathbf{H})$ is at least $q+2$. Therefore LP decoding can correct at least $\left\lfloor\frac{q+2-1}{2}\right\rfloor=\frac{q}{2}$ bit flips.

Let us now show that LP decoding can correct at most $q$ bit flips. Remember that a necessary condition for LP decoding to decode a received log-likelihood vector $\boldsymbol{\lambda}$ to the zero codeword is that $\langle\boldsymbol{\omega}, \boldsymbol{\lambda}\rangle \geqslant 0$ for all $\boldsymbol{\omega} \in \mathcal{K}(\mathbf{H}) .{ }^{15}$ Assume that we are transmitting the zero codeword and that $e$ bit flips happened. Hence $e$ components of $\boldsymbol{\lambda}$ are equal to $-L$ and $n-e$ components of $\boldsymbol{\lambda}$ are equal $+L$. It can easily be checked that the following $\boldsymbol{\omega}$ is in $\mathcal{K}(\mathbf{H})$ : let $\omega_{i} \triangleq 1$ if $\lambda_{i}=-L$ and $\omega_{i} \triangleq 1 / q$ otherwise. For this $\boldsymbol{\omega}$, the condition $\langle\boldsymbol{\omega}, \boldsymbol{\lambda}\rangle \geqslant 0$ translates into $e \cdot(-L)+(n-e) \cdot(1 / q) \cdot(+L) \geqslant 0$, i.e. $e \leqslant \frac{n}{q+1}=\frac{q^{2}+q+1}{q+1}=q+\frac{1}{q+1}$. Because $e$ must be an integer this inequality turns into the inequality $e \leqslant\left\lfloor q+\frac{1}{q+1}\right\rfloor=q$.

Observe that the way we constructed the pseudo-codeword $\boldsymbol{\omega}$ in the proof of Th. 25] can be seen as a generalization of the so-called canonical completion [3, 4, however instead of assigning values according to the graph distance with respect to a single node, we assign values according to the graph distance with respect to the set of nodes where $\lambda_{i}$ is negative. (Note that the Tanner graph of $\mathbf{H}=\mathbf{H}_{\mathrm{PG}(2, q)}$ has a special property: all variable nodes are at graph distance 2 from each other.) Such a generalization of the canonical completion was also used by Haley and Grant [26] for the analysis of their codes.

Corollary 26 Consider the code defined by $\mathbf{H} \triangleq \mathbf{H}_{\mathrm{PG}(2, q)}$. For the BSC, a necessary condition for a minimal pseudo-codeword $\boldsymbol{\omega}$ of $\mathcal{K}(\mathbf{H})$ to be effective of the second kind is that $q+2 \leqslant w_{\mathrm{p}}^{\mathrm{BSC}}(\boldsymbol{\omega}) \leqslant 2 q+2$.

Proof: See Sec. A.2.

For $q=4$ it turns out that $\mathcal{K}\left(\mathbf{H}_{\mathrm{PG}(2,4)}\right)$ has minimal pseudo-codewords with BSC pseudo-weight equal to 12 . (These minimal pseudo-codewords have type $\mathbf{t}$ with $t_{2}=1$, $t_{1}=12, t_{0}=8$, and $t_{\ell}=0$ otherwise.) Cor. 26 clearly shows that these cannot be effective of the second kind for the BSC, since, for $q=4$, any effective minimal pseudo-codeword of the second kind must fulfill $6 \leqslant w_{\mathrm{p}}^{\mathrm{BSC}}(\boldsymbol{\omega}) \leqslant 10$.

Judging from Fig. 1 it also seems - as far as AWGNC and BSC pseudo-weight are comparable - that soft information is quite helpful for the LP decoder when decoding the code $\mathcal{C}_{\mathrm{PG}(2,4)}$ defined by $\mathbf{H}_{\mathrm{PG}(2,4)}$.

We now turn to the BEC. Because $\langle\boldsymbol{\omega}, \boldsymbol{\lambda}\rangle \geqslant 0$ for all minimal pseudo-codewords $\boldsymbol{\omega}$, no minimal pseudo-codeword can be effective of the first kind.

\footnotetext{
${ }^{15}$ Note that this is usually not a sufficient condition for correct decoding, e.g. in the case where ties are resolved randomly.
} 


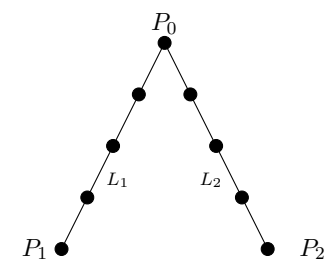

Figure 5: Part of $\mathrm{PG}(2,4)$ discussed in Ex. 29

Theorem 27 Consider data transmission over a BEC using the code defined by $\mathbf{H}$. Let $\boldsymbol{\omega}$ be a minimal pseudo-codeword such that there is an unscaled pseudo-codeword associated to $\boldsymbol{\omega}$ with at least one odd component. Then there exists a non-zero codeword $\mathbf{c}$ such that whenever $\langle\boldsymbol{\omega}, \boldsymbol{\lambda}\rangle=0$ for some $\boldsymbol{\lambda} \in \mathcal{L}_{\mathrm{BEC}}^{(n)}$ then also $\langle\mathbf{c}, \boldsymbol{\lambda}\rangle=0$.

Proof: Let $\boldsymbol{\omega}^{\prime}$ be an unscaled pseudo-codeword that is a positive multiple of $\boldsymbol{\omega}$. By assumption, we can assume that at least one component of $\boldsymbol{\omega}^{\prime}$ is an odd integer. It follows [4] that $\mathbf{c} \triangleq \boldsymbol{\omega}^{\prime}(\bmod 2)$ is a non-zero codeword. Let $\boldsymbol{\lambda} \in \mathcal{L}_{\mathrm{BEC}}^{(n)}$ be such that $\langle\boldsymbol{\omega}, \boldsymbol{\lambda}\rangle=0$. Because $\boldsymbol{\omega}^{\prime}$ is a positive multiple of $\boldsymbol{\omega}$ we must have $\left\langle\boldsymbol{\omega}^{\prime}, \boldsymbol{\lambda}\right\rangle=0$ and because $\operatorname{supp}(\mathbf{c}) \subseteq \operatorname{supp}\left(\boldsymbol{\omega}^{\prime}\right)$ we must have $\langle\mathbf{c}, \boldsymbol{\lambda}\rangle=0$.

Corollary 28 Consider data transmission over a BEC using the code defined by $\mathbf{H}$. Under block-wise $M L$ decoding we define a block error to be the event that there is a tie among at least two codewords. Similarly, under LP decoding we define a block error to be the event that there is a tie among at least two pseudo-codewords. If for all minimal pseudo-codewords there exists an associated pseudo-codeword with at least one odd component then the block error rate of block-wise $M L$ decoding coincides with the block error rate of LP decoding.

By listing all the minimal pseudo-codewords, it can be shown numerically that the condition in Cor. 28 is fulfilled for $\mathbf{H} \triangleq \mathbf{H}_{\mathrm{PG}(2, q)}$ when $q=2$ and $q=4$. It follows that for these two codes block-wise ML and LP decoding yield the same block error rate (under the above definition of block error rate). This corroborates the observations made in 33 , Fig. 1] for $q=4$.

\section{The Structure of Minimal Pseudo-Codewords}

In this section we discuss the geometry of minimal pseudo-codewords. Minimum-weight codewords correspond to point-line configurations in the projective plane that have been studied by several authors. Let us introduce some notation and results from finite geometries, cf. e.g. 9]. A $k$-arc in $\mathrm{PG}(2, q)$ is a set of $k$ points no three of which are collinear. A $k$-arc is complete if it is not contained in a $(k+1)$-arc. The maximum number of points that a $k$-arc can have is denoted by $m(2, q)$, and a $k$-arc with this number of points is called an oval (in the case where $q$ is even this is sometimes also called a hyper-oval). One can show that $m(2, q)=q+2$ for $q$ even and $m(2, q)=q+1$ for $q$ odd. One can make the following two interesting observations for the case $q$ even. Firstly, if two ovals have more than half their points in common, then these two ovals coincide. Secondly, if a $q$-arc is contained in an oval then the number of such ovals is one if $q>2$ and two if $q=2$.

It turns out that in the case $q$ even, the codewords with minimal weight are $q+2$-arcs and therefore ovals. However, whereas the classification of ovals for odd $q$ is simple (they 
all correspond to conics), the ovals for even $q$ are not classified that easily. For even $q$, one says that an oval is regular if it comprises the points of a conic and its nucleus; one can show that for $q=2^{s}$, irregular ovals exist if and only if $s \geqslant 4$. It turns out that the classification for irregular ovals is highly non-trivial. ${ }^{16}$ So, given that even the classification of the codewords of minimal weight is difficult, it is probably hopeless to obtain a complete classification of the minimal codewords and minimal pseudo-codewords of codes defined by $\mathbf{H}_{\mathrm{PG}(2, q)}$, however it is an interesting goal to try to understand as much as possible about the structure of these codewords and pseudo-codewords.

In some recent papers, the structure of codewords of projective-plane-based codes has been discussed by Kashyap and Vardy [29] (that paper talks also about stopping sets in Tanner graphs derived from projective planes), by Justesen et al. 34, 35. and by Laendner and Milenkovic [36] (that paper also talks about trapping sets in Tanner graphs derived from projective planes). Moreover, the minimal-weight codewords of Euclidean-plane-based codes were discussed by Høholdt et al. [37]; also here these configurations are tightly related to ovals. However, because not all ovals are regular, the classification is not that simple also for these codes. ${ }^{17}$

From now on, we will only consider projective planes $\mathrm{PG}(2, q)$ and $q$ will always be even, i.e. a power of two. Before we state our conjecture about the structure of minimal pseudo-codewords, let us first look at an example.

Example 29 Let $q=4$. We can find a minimal pseudo-codeword $\boldsymbol{\omega}$ whose type $\mathbf{t}$ is $t_{0}=8$, $t_{1}=8, t_{2}=5$, and $t_{\ell}=0$ otherwise. This pseudo-codeword can be obtained using a procedure similar to the one used in Ex. 21. Firstly, on has to add two vectors $\mathbf{x}^{(1)}$ and $\mathbf{x}^{(2)}$ of weight 6 whose supports overlap in two positions. This yields a pseudo-codeword $\tilde{\boldsymbol{\omega}}$ of type $\tilde{\mathbf{t}}$ with $\tilde{t}_{0}=11, \tilde{t}_{1}=8, \tilde{t}_{2}=2$, and $\tilde{t}_{\ell}=0$ otherwise. Secondly, in order to obtain a minimal pseudo-codeword, one has to switch three zeros (that were appropriately chosen) into three twos.

Let us analyze this procedure. Since a minimal pseudo-codeword corresponds to an edge of the fundamental cone, it is clear that the inequalities in (4) and (5) that are fulfilled with equality must form a system of linear equations of rank $21-1=20$. We start with two minimal codewords $\mathbf{x}^{(1)}$ and $\mathbf{x}^{(2)}$ that each yield a system of linear equations of rank $21-1=20$. These two codewords have been chosen such that their sum $\tilde{\boldsymbol{\omega}}$ yields a system of linear equations of rank $21-2=19$.

To find the three zeros that we have to switch, we proceed as follows. It turns out that in the projective plane $\mathrm{PG}(2,4)$ there are two lines, say $L_{1}$ and $L_{2}$, such that all the entries of $\tilde{\boldsymbol{\omega}}$ that correspond to the points on these two lines are zero. Let $P_{0}$ be the intersection point of these two lines, cf. Fig. 15. There exists a point $P_{1}$ on $L_{1}$ and a point $P_{2}$ on $L_{2}$ such that modifying $\tilde{\boldsymbol{\omega}}$ by assigning them the same value $\alpha \geqslant 0$ yields a vector in the fundamental cone, as long as $\alpha$ is not too large. In fact, for $\alpha>2$ the vector is outside the fundamental cone, and for $\alpha=2$ it yields a vector that is a pseudo-codeword and that yields a system of equations of rank $21-1=20$, i.e. it is a minimal pseudo-codeword.

Conjecture 30 For the Tanner graph defined by $\mathbf{H}_{\mathrm{PG}(2, q)}$ every minimal pseudo-codeword is a sum of a few minimal pseudo-codewords with a change of a few low-value components

\footnotetext{
${ }^{16}$ According to [9] Th. 8.35], there are precisely two projectively distinct ovals in $\mathrm{PG}(2,16)$, the so-called regular oval $\mathcal{D}\left(T^{2}\right)$ and the so-called oval $\mathcal{O}_{0}=\mathcal{D}\left(F_{0}\right)$. Moreover, according to [9. Th. 8.36], there are precisely six projectively distinct ovals in $\mathrm{PG}(2,32)$.

${ }^{17}$ Note that the remark "It is also known that an oval in EG $(2, q)$ consists of a conic and a nucleus" (which would imply that they are regular) in [37 Sec. 2] is wrong in general [38].
} 
such that they become the large components in the equations associated to the lines that pass through them.

Hence, to find minimal pseudo-codewords, we have to take sums of two minimal pseudocodewords that give rank $n-2$ (if possible; lower otherwise) and change few components that are "not significant" into a "significant" ones. We call a component significant if it is the sum of the other components that belong to a line passing through the point, for most of such lines.

The following conjecture is based on our observations (for small $q$ ) that among the minimal pseudo-codewords the ones with only zeros, ones, and twos yield non-codeword minimal pseudo-codewords of smallest weight. A positive answer to this conjecture would result in a much better understanding of the minimal pseudo-codewords in general and of the AWGNC pseudo-weight spectrum gap, in particular.

Conjecture 31 Let $\mathbf{H} \triangleq \mathbf{H}_{\mathrm{PG}(2, q)}$ and consider the pseudo-codewords that have minimal AWGNC pseudo-weight among all minimal pseudo-codewords that are not multiples of minimal codewords. We conjecture that the type $\mathbf{t}$ of these pseudo-codewords is such that $t_{0}$ is non-negative, $t_{1}$ is positive, $t_{2}$ is positive, and $t_{\ell}=0$ otherwise. (If this conjecture is not true, find the the smallest $\tilde{\ell}$ such that these pseudo-codewords have type $\mathbf{t}$ with $t_{\ell} \geqslant 0$ for $\ell \in\{0,1, \ldots, \tilde{\ell}\}$ and $t_{\ell}=0$ otherwise.)

\section{Conclusions}

We have investigated the minimal pseudo-codewords of some codes whose Tanner graphs are derived from projective and Euclidean planes and we have introduced the notion of a pseudo-weight spectrum gap for a parity-check matrix, a concept which is certainly worthwhile to be further explored. Although our numerical results are for codes of modest length, to the best of our knowledge this is the first study that tries to analytically quantify the behavior of $\mathrm{PG}(2, q)$ - and $\mathrm{EG}(2, q)$-based binary linear codes under LP and iterative decoding. Extending these results to somewhat longer codes has the potential to explain many experimental observations made in the past. Moreover, we have obtained a clearer picture about the structure of the minimal pseudo-codewords of the Tanner graphs under investigation, nevertheless more work is required to get a sufficiently tight characterization of them.

We also introduced the notion of the effectiveness of a minimal pseudo-codeword and we saw that in the case of non-AWGNC channels there are minimal pseudo-codewords that are not effective (of the first kind or of the second kind). Interestingly, in that context we were able to use the canonical completion, a tool that so far has been very useful for characterizing families of $\left(w_{\text {col }}, w_{\text {row }}\right)$-regular LDPC codes, with $w_{\text {col }}, w_{\text {row }}$ bounded when the block length goes to infinity, i.e. code families where the Tanner graph diameter grows with the block length. (This is in contrast to the $\mathrm{PG}(2, q)$-based Tanner graphs which have girth 6 and diameter 3 , independently of $q$ ).

\section{A Proofs}

We include here two of the larger proofs of this paper. 


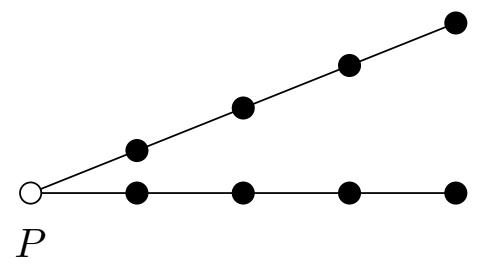

Figure 6: Part of $\operatorname{PG}(2, q)$ showing the relevant part of a codeword with Hamming weight 2q. (Here for $q=4$.)

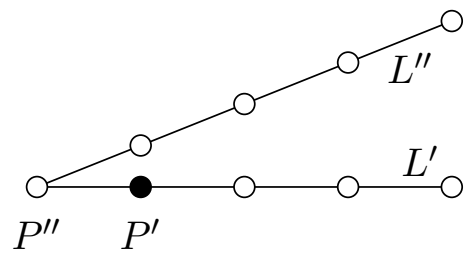

Figure 7: Part of $\operatorname{PG}(2, q)$ showing the points $P^{\prime}$ and $P^{\prime \prime}$ and the lines $L^{\prime}$ and $L^{\prime \prime}$ that were used in proof of Th. 13. (Here for $q=4$.)

\section{A.1 Proof of Theorem 13}

Let $\mathbf{s}^{\top} \triangleq \mathbf{H} \cdot \boldsymbol{\omega}^{\top}$ (in $\mathbb{R}$ ) and let $\mathcal{J}_{\text {odd }} \triangleq\left\{j \in \mathcal{J} \mid s_{j}\right.$ is odd $\}$ be the set of all rows of $\mathbf{H}$ where the corresponding entry in $\mathbf{s}$ is an odd integer. If $\boldsymbol{\omega}$ were a codeword then all entries of $\mathbf{s}$ would be non-negative even integers. However, because $\boldsymbol{\omega}$ is assumed to be a non-codeword, $\left|\mathcal{J}_{\text {odd }}\right| \geqslant 1$. Note moreover that because $\operatorname{supp}(\boldsymbol{\omega})$ is a stopping set, we must have $s_{j} \neq 1$ for all $j \in \mathcal{J}$.

The proof proceeds as follows. First we will show that for any non-codeword $\boldsymbol{\omega}$ we must have $\left|\mathcal{J}_{\text {odd }}\right| \geqslant q+1$. Secondly, we will show that this lower bound on $\left|\mathcal{J}_{\text {odd }}\right|$ implies the lower bound mentioned in the theorem.

So, let us show that $\left|\mathcal{J}_{\text {odd }}\right| \geqslant q+1$ for any non-codeword $\boldsymbol{\omega}$. The proof is by contradiction, i.e. if $\mathcal{J}_{\text {odd }}$ has $\left|\mathcal{J}_{\text {odd }}\right| \leqslant q$, we will show that there exists a $j \in \mathcal{J}_{\text {odd }}$ such that the value of $s_{j}$ must be an even integer. To that end, it turns out to be useful to reverse the usual interpretation of the columns and rows of $\mathbf{H}$ : the columns will correspond to lines and the rows will correspond to points of $\operatorname{PG}(2, q)$. With this, there is a one-to-one relationship between the points of $\operatorname{PG}(2, q)$ and the entries of $\mathbf{s}$.

Before we proceed, consider Fig. [6 that shows two lines in $\operatorname{PG}(2, q)$ and the points on them (here for $q=4$ ). Letting $\mathbf{x} \in \mathbb{R}^{n}$ be a vector where the entries corresponding to black dots equal 1 and the other entries equal 0 , one can easily verify that $\mathbf{x} \cdot \mathbf{H}=\mathbf{0}(\bmod 2)$, i.e. $\mathbf{x}$ is a codeword of the code with parity-check matrix $\mathbf{H}^{\top}$.

Now, choose any $j \in \mathcal{J}_{\text {odd }}$, consider Fig. [7, and let $P^{\prime}$ correspond to the $j$-th row of $\mathbf{H}$. Because $\left|\mathcal{J}_{\text {odd }}\right| \leqslant q$, it is possible to choose a line $L^{\prime}$ through $P^{\prime}$ such that all points on it (except for $P^{\prime}$ ) have an even $s_{j}$. On this line it is then possible to choose (again because $\left.\left|\mathcal{J}_{\text {odd }}\right| \leqslant q\right)$ a $P^{\prime \prime} \neq P^{\prime}$ such that there is a line $L^{\prime \prime}$ through it such that all points on it have an even $s_{j}$. Let $\mathrm{x} \in \mathbb{R}^{n}$ be a vector where the non-zero entries corresponding to the points on $L^{\prime}$ and $L^{\prime \prime}$ (except for $P^{\prime \prime}$ ) equal 1 and the other entries equal 0. Because of the considerations in the paragraph above, it is clear that $\mathbf{x} \cdot \mathbf{H}=\mathbf{0}(\bmod 2)$, i.e. all entries in $\mathbf{x} \cdot \mathbf{H}$ are even integers. This implies that $\mathbf{x} \cdot \mathbf{s}^{\top}=\mathbf{x} \cdot\left(\mathbf{H} \cdot \boldsymbol{\omega}^{\top}\right)=(\mathbf{x} \cdot \mathbf{H}) \cdot \boldsymbol{\omega}^{\top}$ must be an 
even integer. This is a contradiction, because of the way we have chosen $P^{\prime}, L^{\prime}, P^{\prime \prime}$, and $L^{\prime \prime}$, the inner product $\mathbf{x} \cdot \mathbf{s}^{\top}$ must be an odd integer.

Let us now prove that the lower bound $\left|\mathcal{J}_{\text {odd }}\right| \geqslant q+1$ on $\left|\mathcal{J}_{\text {odd }}\right|$ implies the lower bound on $w_{\mathrm{p}}(\boldsymbol{\omega})$ mentioned in the theorem. Because of the special properties of $\mathbf{H}$ we have $\mathbf{H}^{\top} \mathbf{H}=q \mathbf{I}+\mathbf{J}$, where $\mathbf{I}$ is the identity matrix of size $n \times n$ and where $\mathbf{J}$ is the all-ones matrix of size $n \times n$. Then $\|\mathbf{s}\|_{2}^{2}=\mathbf{s s}^{\top}=\boldsymbol{\omega} \mathbf{H}^{\top} \mathbf{H} \boldsymbol{\omega}^{\top}=q \boldsymbol{\omega} \mathbf{I} \boldsymbol{\omega}^{\top}+\boldsymbol{\omega} \mathbf{J} \boldsymbol{\omega}^{\top}=q\|\boldsymbol{\omega}\|_{2}^{2}+\|\boldsymbol{\omega}\|_{1}^{2}=q t_{1}+t_{1}^{2}$. On the other hand,

$$
\|\mathbf{s}\|_{2}^{2}=\sum_{j \in \mathcal{J}} s_{j}^{2}=\sum_{j \in \mathcal{J}}\left(\sum_{i \in \mathcal{I}_{j}} \omega_{i}\right)^{2}=\sum_{j \in \mathcal{J}} \sum_{i \in \mathcal{I}_{j}} \omega_{i} \sum_{i^{\prime} \in \mathcal{I}_{j}} \omega_{i^{\prime}} .
$$

We would like to find a lower bound on $\|\mathbf{s}\|_{2}^{2}$. If $j \in \mathcal{J} \backslash \mathcal{J}_{\text {odd }}$ then we use $\sum_{i^{\prime} \in \mathcal{I}_{j}} \omega_{i^{\prime}} \geqslant 2 \omega_{i}$, for all $i$ in $\mathcal{I}_{j}$ (which is implied by (4)), otherwise we use $\sum_{i^{\prime} \in \mathcal{I}_{j}} \omega_{i^{\prime}} \geqslant 2 \omega_{i}+1$ (which also follows from (44), together with the observation that $s_{j}=\sum_{i^{\prime} \in \mathcal{I}_{j}} \omega_{i^{\prime}} \geqslant 3$ ):

$$
\begin{aligned}
\|\mathbf{s}\|_{2}^{2} & =\sum_{j \in \mathcal{J}} \sum_{i \in \mathcal{I}_{j}} \omega_{i} \sum_{i^{\prime} \in \mathcal{I}_{j}} \omega_{i^{\prime}} \geqslant 2 \sum_{j \in \mathcal{J}} \sum_{i \in \mathcal{I}_{j}} \omega_{i}^{2}+\sum_{j \in \mathcal{J}_{\text {odd }}} \sum_{i \in \mathcal{I}_{j}} \omega_{i}=2 \sum_{i \in \mathcal{I}} \sum_{j \in \mathcal{J}_{i}} \omega_{i}^{2}+\sum_{j \in \mathcal{J}_{\text {odd }}} \sum_{i \in \mathcal{I}_{j}} \omega_{i} \\
& =2(q+1)\|\boldsymbol{\omega}\|_{2}^{2}+\sum_{j \in \mathcal{J}_{\text {odd }}} \sum_{i \in \mathcal{I}_{j}} \omega_{i} \geqslant 2(q+1)\|\boldsymbol{\omega}\|_{2}^{2}+3\left|\mathcal{J}_{\text {odd }}\right|=2(q+1) t_{1}+3\left|\mathcal{J}_{\text {odd }}\right| .
\end{aligned}
$$

Combining the above results we obtain $q t_{1}+t_{1}^{2}=\|\mathbf{s}\|_{2}^{2} \geqslant 2(q+1) t_{1}+3\left|\mathcal{J}_{\text {odd }}\right|$, or, equivalently, $t_{1}^{2}-(q+2) t_{1}-3\left|\mathcal{J}_{\text {odd }}\right| \geqslant 0$. It follows that $t_{1} \geqslant \frac{q}{2}+1+\frac{1}{2} \sqrt{(q+2)^{2}+12\left|\mathcal{J}_{\text {odd }}\right|} \cdot{ }^{18}$ Inserting the lower bound $\left|\mathcal{J}_{\text {odd }}\right| \geqslant q+1$, we obtain

$$
t_{1} \geqslant \frac{q}{2}+1+\frac{1}{2} \sqrt{q^{2}+16 q+16}
$$

Because $w_{\mathrm{p}}(\boldsymbol{\omega})=t_{1}$ and because $t_{1}$ is an integer, the final result follows.

We conclude with two remarks:

- More sophisticated considerations might lead to better lower bounds on $w_{\mathrm{p}}(\boldsymbol{\omega})$; however, note that $\left|\mathcal{J}_{\text {odd }}\right| \geqslant q+1$ is the best lower bound that can be given on the size of $\mathcal{J}_{\text {odd }}$ without additional information about the set. Namely, if $\mathcal{J}_{\text {odd }}$ happens to be equal to $\mathcal{J}_{i}$ for some $i \in \mathcal{I}$ then $\left|\mathcal{J}_{\text {odd }}\right|=q+1$ and no row in $\mathbf{H}$ corresponding to an entry in $\mathcal{J}_{\text {odd }}$ can be expressed as a linear combination of rows corresponding to entries in $\mathcal{J} \backslash \mathcal{J}_{\text {odd }}$.

- The bounding techniques used in the second part of the proof were inspired by the bounding techniques that were used in [24], which in turn were generalizations of [39].

\section{A.2 Proof of Corollary 26}

Let $\boldsymbol{\omega}$ be a minimal pseudo-codeword with $w_{\mathrm{p}}^{\mathrm{BSC}}(\boldsymbol{\omega})>2 q+2$. The proof is by contradiction, i.e. we will assume that $\boldsymbol{\omega}$ is effective of the second kind and then we will show that for any $\boldsymbol{\lambda} \in \mathcal{L}_{\mathrm{BSC}}^{(n)}$ with $\langle\boldsymbol{\omega}, \boldsymbol{\lambda}\rangle \leqslant 0$ there exists a minimal pseudo-codeword $\boldsymbol{\omega}^{\prime \prime} \neq \boldsymbol{\omega}$ such that $\left\langle\boldsymbol{\omega}^{\prime \prime}, \boldsymbol{\lambda}\right\rangle<0$.

Assume that we are transmitting the zero codeword and that bit flips happened at positions $\mathcal{E}$. Hence $|\mathcal{E}|$ components of $\boldsymbol{\lambda}$ are equal to $-L$ and $n-|\mathcal{E}|$ components of $\boldsymbol{\lambda}$ are

\footnotetext{
${ }^{18} t_{1} \leqslant \frac{q}{2}+1-\frac{1}{2} \sqrt{(q+2)^{2}+12\left|\mathcal{J}_{\text {odd }}\right|}$ is not possible because we know that $t_{1} \geqslant q+2$.
} 
equal $+L$. Assume that $\langle\boldsymbol{\omega}, \boldsymbol{\lambda}\rangle \leqslant 0$. From the definition of the BSC pseudo-weight it follows that $|\mathcal{E}|>q+1$, i.e. $|\mathcal{E}| \geqslant q+2$.

Choose a sub-pattern $\mathcal{E}^{\prime} \subset \mathcal{E}$ of bit-flips with $\left|\mathcal{E}^{\prime}\right|=q+1$ and define the corresponding $\boldsymbol{\lambda}^{\prime}$. From the definition of the BSC pseudo-weight it follows that $\left\langle\boldsymbol{\omega}, \boldsymbol{\lambda}^{\prime}\right\rangle>0$ (otherwise $\left.w_{\mathrm{p}}^{\mathrm{BSC}}(\boldsymbol{\omega}) \leqslant 2 q+2\right)$.

Similarly to the proof of Theorem [25] we can construct a pseudo-codeword $\boldsymbol{\omega}^{\prime}$ based on $\mathcal{E}^{\prime}$ such that $\boldsymbol{\omega}_{i}^{\prime} \triangleq 1$ if $i \in \mathcal{E}^{\prime}$ and $\boldsymbol{\omega}_{i}^{\prime} \triangleq 1 / q$ otherwise. This pseudo-codeword has the property that $\left\langle\boldsymbol{\omega}^{\prime}, \boldsymbol{\lambda}^{\prime}\right\rangle=\left|\mathcal{E}^{\prime}\right| \cdot(-L)+\left(n-\left|\mathcal{E}^{\prime}\right|\right) \cdot(1 / q) \cdot(+L)=-L<0$. Let $\left\{\boldsymbol{\omega}^{(\ell)}\right\}_{\ell}$ be the set of all minimal pseudo-codewords. Then $\boldsymbol{\omega}^{\prime}=\sum_{\ell} \alpha_{\ell} \boldsymbol{\omega}^{(\ell)}$ for some choice of $\left\{\alpha_{\ell}\right\}_{\ell}$ where all $\alpha_{\ell}$ are non-negative. Therefore, there exists at least one minimal pseudo-codeword, say $\boldsymbol{\omega}^{\prime \prime}$, such that $\left\langle\boldsymbol{\omega}^{\prime \prime}, \boldsymbol{\lambda}^{\prime}\right\rangle<0$. (It is clear that $\boldsymbol{\omega}^{\prime \prime} \neq \boldsymbol{\omega}$.)

Because $\boldsymbol{\omega}^{\prime \prime} \geqslant \mathbf{0}$, it is easy to see that $\left\langle\boldsymbol{\omega}^{\prime \prime}, \boldsymbol{\lambda}\right\rangle \leqslant\left\langle\boldsymbol{\omega}^{\prime \prime}, \boldsymbol{\lambda}^{\prime}\right\rangle$. This implies that $\left\langle\boldsymbol{\omega}^{\prime \prime}, \boldsymbol{\lambda}\right\rangle<0$, which is the promised contradiction.

\section{References}

[1] J. Feldman, Decoding Error-Correcting Codes via Linear Programming. PhD thesis, Massachusetts Institute of Technology, Cambridge, MA, 2003. Available online under http://www. columbia.edu/ jf2189/pubs.html.

[2] J. Feldman, M. J. Wainwright, and D. R. Karger, "Using linear programming to decode binary linear codes," IEEE Trans. on Inform. Theory, vol. IT-51, no. 3, pp. 954-972, 2005.

[3] R. Koetter and P. O. Vontobel, "Graph covers and iterative decoding of finite-length codes," in Proc. 3rd Intern. Symp. on Turbo Codes and Related Topics, (Brest, France), pp. 75-82, Sept. 1-5 2003.

[4] P. O. Vontobel and R. Koetter, "Graph-cover decoding and finite-length analysis of message-passing iterative decoding of LDPC codes," submitted to IEEE Trans. Inform. Theory, available online under http://www.arxiv.org/abs/cs.IT/0512078, Dec. 2005.

[5] P. O. Vontobel and R. Koetter, "On the relationship between linear programming decoding and min-sum algorithm decoding," in Proc. Intern. Symp. on Inform. Theory and its Applications (ISITA), (Parma, Italy), pp. 991-996, Oct. 10-13 2004.

[6] R. Lucas, M. Fossorier, Y. Kou, and S. Lin, "Iterative decoding of one-step majority logic decodable codes based on belief propagation," IEEE Trans. on Comm., vol. COM48, pp. 931-937, June 2000.

[7] Y. Kou, S. Lin, and M. P. C. Fossorier, "Low-density parity-check codes based on finite geometries: a rediscovery and new results," IEEE Trans. on Inform. Theory, vol. IT-47, pp. 2711-2736, Nov. 2001.

[8] L. M. Batten, Combinatorics of Finite Geometries. Cambridge: Cambridge University Press, second ed., 1997.

[9] J. W. P. Hirschfeld, Projective Geometries over Finite Fields. Oxford Mathematical Monographs, New York: The Clarendon Press and Oxford University Press, second ed., 1998. 
[10] R. M. Tanner, "A recursive approach to low-complexity codes," IEEE Trans. on Inform. Theory, vol. IT-27, pp. 533-547, Sept. 1981.

[11] T. J. Richardson and R. L. Urbanke, "The capacity of low-density parity-check codes under message-passing decoding," IEEE Trans. on Inform. Theory, vol. IT-47, no. 2, pp. 599-618, 2001.

[12] E. Agrell, "Voronoŭ regions for binary linear block codes," IEEE Trans. on Inform. Theory, vol. IT-42, no. 1, pp. 310-316, 1996.

[13] M. M. Deza and M. Laurent, Geometry of Cuts and Metrics, vol. 15 of Algorithms and Combinatorics. Berlin: Springer-Verlag, 1997.

[14] T. Y. Hwang, "Decoding linear block codes for minimizing word error rate," IEEE Trans. on Inform. Theory, vol. IT-25, no. 6, pp. 733-737, 1979.

[15] A. Ashikhmin and A. Barg, "Minimal vectors in linear codes," IEEE Trans. on Inform. Theory, vol. IT-44, no. 5, pp. 2010-2017, 1998.

[16] A. Ashikhmin, A. Barg, G. Cohen, and L. Huguet, "Variations on minimal codewords in linear codes," in Applied Algebra, Algebraic Algorithms and Error-Correcting Codes (Paris, 1995), vol. 948 of Lecture Notes in Comput. Sci., pp. 96-105, Berlin: Springer, 1995.

[17] J. L. Massey, "Minimal codewords and secret sharing," in Proc. 6th Joint SwedishRussian Int. Workshop on Info. Theory, (Mölle, Sweden), pp. 276-279, Aug. 22-27 1993.

[18] Y. Borissov, N. Manev, and S. Nikova, "On the non-minimal codewords in the binary Reed-Muller code," in Proc. IEEE Intern. Symp. on Inform. Theory, (Washington, D.C., USA), p. 39, June 24-29 2001.

[19] D. Bertsimas and J. N. Tsitsiklis, Linear Optimization. Belmont, MA: Athena Scientific, 1997.

[20] R. Koetter, W.-C. W. Li, P. O. Vontobel, and J. L. Walker, "Pseudo-codewords of cycle codes via zeta functions," in Proc. IEEE Inform. Theory Workshop, (San Antonio, TX, USA), pp. 7-12, Oct. 24-29 2004. Available online under http://www. arxiv.org/abs/cs.IT/0502033.

[21] S. Boyd and L. Vandenberghe, Convex Optimization. Cambridge, UK: Cambridge University Press, 2004.

[22] N. Wiberg, Codes and Decoding on General Graphs. PhD thesis, Linköping University, Sweden, 1996.

[23] G. D. Forney, Jr., R. Koetter, F. R. Kschischang, and A. Reznik, "On the effective weights of pseudocodewords for codes defined on graphs with cycles," in Codes, Systems, and Graphical Models (Minneapolis, MN, 1999) (B. Marcus and J. Rosenthal, eds.), vol. 123 of IMA Vol. Math. Appl., pp. 101-112, Springer Verlag, New York, Inc., 2001. 
[24] P. O. Vontobel and R. Koetter, "Lower bounds on the minimum pseudo-weight of linear codes," in Proc. IEEE Intern. Symp. on Inform. Theory, (Chicago, IL, USA), p. 70, June 27-July 22004.

[25] D. J. C. MacKay and M. C. Davey, "Evaluation of Gallager codes for short block length and high rate applications," in Codes, Systems, and Graphical Models (Minneapolis, $M N$, 1999) (B. Marcus and J. Rosenthal, eds.), pp. 113-130, Springer Verlag, New York, Inc., 2001.

[26] D. Haley and A. Grant, "Improved reversible LDPC codes," in Proc. IEEE Intern. Symp. on Inform. Theory, (Adelaide, Australia), pp. 1367-1371, Sep. 4-9 2005.

[27] G. E. Séguin, "A lower bound on the error probability for signals in white Gaussian noise," IEEE Trans. on Inform. Theory, vol. IT-44, no. 7, pp. 3168-3175, 1998.

[28] D. Avis, "lrs: A revised implementation of the reverse search vertex enumeration algorithm," in Polytopes - Combinatorics and Computation (G. Kalai and G. M. Ziegler, eds.), pp. 177-198, Birkhäuser-Verlag, 2000. Programs are available online under http://cgm.cs.mcgill.ca/ avis/C/lrs.html.

[29] N. Kashyap and A. Vardy, "Stopping sets in codes from designs," in Proc. IEEE Intern. Symp. on Inform. Theory, (Pacifico Yokohama, Japan), p. 122, June 29 - July 42003. Extended version available online under http://www. mast.queensu.ca/ ${ }^{\sim}$ nkashyap.

[30] M. Wauer, "LDPC codes based on projective geometries," Master's thesis, Dept. of Math. and Stat., San Diego State University, San Diego, CA, USA, 2005.

[31] C. Kelley and D. Sridhara, "Pseudocodewords of Tanner graphs," submitted to IEEE Trans. Inform. Theory, available online under http://www. arxiv.org/abs/cs. IT/0504013, Apr. 2005.

[32] P. O. Vontobel and R. Smarandache, "On minimal pseudo-codewords of Tanner graphs from projective planes," in Proc. 43rd Allerton Conf. on Communications, Control, and Computing, (Allerton House, Monticello, Illinois, USA), Sep. 28-30 2005. Available online under http://www.arxiv.org/abs/cs.IT/0510043.

[33] M. Zhu and K. M. Chugg, "Lower bounds on stopping distance of linear codes and their applications," in Proc. 43rd Allerton Conf. on Communications, Control, and Computing, (Allerton House, Monticello, Illinois, USA), Sep. 28-30 2005.

[34] J. Justesen, T. Høholdt, and J. Hjaltason, "Complete ML decoding for the $(73,45)$ PG code," in Proc. 43rd Allerton Conf. on Communications, Control, and Computing, (Allerton House, Monticello, Illinois, USA), Sep. 28-30 2005.

[35] J. Justesen, T. Høholdt, and J. Hjaltason, "Iterative list decoding," submitted to IEEE Trans. Inform. Theory, Nov. 2005.

[36] S. Laendner and O. Milenkovic, "Algorithmic and combinatorial analysis of trapping sets in structured LDPC codes," in Proc. 2005 International Conference on Wireless Networks, Communications, and Mobile Computing (Wirelesscom 2005), (Maui, HI, USA), Jun. 13-16 2005. 
[37] T. Høholdt, J. Justesen, and B. Jonsson, "Euclidean geometry codes, minimum weight words and decodable error-patterns using bit-flipping," in Proc. IEEE Intern. Symp. on Inform. Theory, (Adelaide, Australia), pp. 2171-2173, Sep. 4-9 2005.

[38] T. Høholdt, personal communication, Dec. 2005.

[39] R. M. Tanner, "Minimum-distance bounds by graph analysis," IEEE Trans. on Inform. Theory, vol. IT-47, no. 2, pp. 808-821, 2001. 